\title{
Molecular dynamics modeling of PPTA crystallite mechanical properties in the presence of defects
}

\author{
Brian MERCER ${ }^{1,2}$, Edward ZYWICZ ${ }^{*}$ and Panayiotis PAPADOPOULOS ${ }^{1, \dagger}$ \\ ${ }^{1}$ Department of Mechanical Engineering, University of California, Berkeley, CA, USA \\ ${ }^{2}$ Lawrence Livermore National Laboratory, Livermore, CA, USA
}

\section{Table of contents}

1 Introduction and background 3

2 Methods 5

2.1 Force field selection . . . . . . . . . . . . . . . . . . 5

2.2 Molecular model . . . . . . . . . . . . . . . . . . . . . . 6

3 Force field validation $\quad 9$

3.1 Unit cell geometry . . . . . . . . . . . . . . . . . . . . . . . 9 9

3.2 Calculation of elastic moduli f . . . . . . . . . . . . . . . . . . . 11

3.3 Molecular geometry changes with axial stretch . . . . . . . . . . . . 13

4 Zone of influence of chain-end defects in the PPTA crystallite 16

4.1 Influence of a single isolated defect . . . . . . . . . . . . . . 16

4.2 Influence of multiple defects . . . . . . . . . . . . . . . . . . . . . . . . . 19

$5 \quad$ Failure of PPTA crystallites under constant strain-rate loading 21

5.1 Failure of defect-free crystallites . . . . . . . . . . . . . . . . . 21

5.2 Failure in crystallites containing chain-end defects . . . . . . . . . 23

6 Role of defects in crystallite modulus $\quad 26$

7 Summary and conclusions $\quad 29$

$\begin{array}{ll}\text { References } & 30\end{array}$

Appendix $\quad 37$

A Derivation of elastic moduli for crystallite with non-interacting defects . . . 37

\begin{abstract}
The mechanical properties of PPTA crystallites, the fundamental building blocks of aramid polymer fibers such as Kevlar ${ }^{\circledR}$ and Twaron ${ }^{\circledR}$, are studied here using molecular dynamics simulations. The REAXFF interatomic potential is employed to study crystallite failure via covalent and hydrogen bond rupture in constant strain-rate tensile loading simulations. Emphasis is placed on analyzing how chain-end defects in the crystallite influence its mechanical response and fracture strength. Chain-end defects are found to affect the behavior of nearby chains in a region of the PPTA crystallite that is small relative to the typical crystallite size in manufactured aramid fibers. The central $\mathrm{C}-\mathrm{N}$ bond along the backbone chain is identified as the weakest in the PPTA polymer chain backbone in dynamic strain-to-failure simulations of the
\end{abstract}

*zywicz1@llnl.gov

†panos@berkeley.edu

(C) 2017. This manuscript version is made available under the Elsevier user license http://www.elsevier.com/open-access/userlicense/1.0/ 
crystallite. It is found that clustering of chain-ends leads to reduced crystallite strength and crystallite failure via hydrogen bond rupture and chain sliding, whereas randomly scattered defects impact the strength less and failure is by covalent bond rupture and chain scission. The axial crystallite modulus increases with increasing chain length and is independent of chain-end defect locations. On the basis of these findings, a theoretical model is proposed to predict the axial modulus as a function of chain length.

Keywords: Kevlar ${ }^{\circledR}$; aramid fibers; elastic modulus; defects; molecular dynamics; reactive potentials 


\section{Introduction and background}

Synthetic polymer fibers play a key role in many engineering applications including fiber-reinforced composites, sporting equipment, and woven fabrics. Among the strongest of man-made polymer fibers are aromatic polyamides, or aramid fibers, the most well-known examples of which are the trademarked fibers Kevlar ${ }^{\circledR}$ and Twaron $^{\circledR}$. Aramid fibers consist of long-chain molecules made from the monomer PPTA. Northolt et al. [1] showed through X-ray diffraction that the microstructure of PPTA fibers consists of a highly crystalline arrangement of extended PPTA chains aligned along the axis of the fiber. The orthogonal crystalline unit cell of the chains is depicted in Figure 1, where it can be seen that hydrogen bonding between adjacent chains occurs in the $b$-direction. Studies by Dobb et al. [2-4] showed that PPTA chains are organized into hydrogen-bonded sheets, and that these sheets are arranged radially within the fiber. Further investigation of fiber microstructure [57] revealed that PPTA chains are organized into crystallites, which are regions of highly crystalline arrangements of PPTA chains separated by periodically-repeating defect planes along the fiber axis, as depicted in Figure 2. These studies do not exactly agree on all the details of the fiber morphology, yet collectively show that the fiber microstructure comprises collections of crystallites between 60 and $600 \mathrm{~nm}$ in diameter made from PPTA chains of about $200 \mathrm{~nm}$ in length, with periodic defect planes every 20-40 $\mathrm{nm}$ along the fiber axis consisting of collections of chain-ends. Additionally, the fibers exhibit a 0.1-1.0 nm-thick skin region where chain-ends are distributed randomly along the fiber axis rather than being organized into defect planes.

Clearly, the mechanical properties of a given aramid fiber depend on the details of the fiber microstructure, which can vary greatly with the fiber manufacturing process. Many models have been developed which use known fiber microstructural properties to predict various mechanical properties of interest. For example, Northolt et al. [810] use knowledge of the elastic properties of crystalline PPTA and the distribution of chain orientation angles relative to the fiber axis to predict the modulus of a given fiber. Rao et al. [11] derived an experiment-based model which identifies the chain orientation distribution and the paracrystalline parameter (a measure of the crystallinity of the fiber microstructure) as the two primary factors that influence 
the fiber modulus. These models work well for predicting the axial fiber modulus for most grades of Kevlar fibers, and demonstrate how knowledge of the microstructural details of the fiber can be used to obtain predictions of fiber mechanical behavior.

Molecular dynamics (MD) is a useful tool in studying the influence of microstructure on macroscopic fiber behavior. It is powerful due to its ability to explicitly model atomic interactions in a material, and can account for the influence of defects and other structural irregularities on its overall macroscopic properties. MD has been previously used to study the material properties of crystalline PPTA, and simulations have been performed to predict the ideal molecular geometry and chain conformations $[12,13]$, elastic moduli $[14,15]$, and compressive failure due to chain buckling [16]. The more recent work of Grujicic et al. [17-22], which represents the most comprehensive set of studies to date, focused on modeling various types of defects and impurities, as well as static tensile, torsion, and compressional loading, and the resulting elastic moduli and axial strength of PPTA crystals.

In this work, MD simulations are used to study the molecular-level behavior and overall mechanical response of defect-containing PPTA crystallites subjected to dynamic tensile loading along the fiber axis. The crystallite is chosen as the subject of study since it is the fundamental building block of PPTA fibers and its length-scale is compatible with MD modeling limitations with respect to system size. Constant strain-rate tensile loading simulations of PPTA crystallites are performed to examine their behavior under conditions mimicking those experienced by individual fibers in ballistic fabrics. The simulations presented here place emphasis on understanding how individual chain-end defects influence local polymer chain mechanics and lead to the degradation of fracture strength and stiffness in the crystallite. By using the reactive bond order force field REAxFF [23-26] to model atomic interactions, it is possible to study crystallite failure mechanisms involving primary (covalent) and/or secondary (hydrogen) bond rupture, and how those mechanisms depend on the distribution of defects within the crystallite. It is found, for example, that when defects are clustered closely together, crystallite failure is dominated by inter-chain slippage and hydrogen bond failure, but when defects are scattered more uniformly throughout the crystallite, crystallite failure is dominated by primary bond rupture along PPTA chain backbones. In particular, these simulations identify the central C$\mathrm{N}$ bond in the amide linkage along the PPTA chain backbone as the weakest covalent 
bond in the chain, and therefore the agent that controls the overall crystallite strength in the absence of defect clusters. The combination of dynamic loading simulations and a detailed study of the polymer chain mechanics and failure behavior in the presence of defects represents an important contribution to the MD literature on PPTA and offers key insights into the crystallite mechanical behavior and the role of defects in determining that behavior, thereby enhancing the understanding of the overall constitutive behavior of single aramid fibers.

The remainder of this article is organized as follows. Section 2 presents the details of the MD simulation methods used here, including generation of atomistic PPTA crystallite models, interatomic force field selection, and implementational details. Section 3 documents the process of validating the choice of REAxFF parameterization [27] used to perform the PPTA simulations. Section 4 analyzes how the introduction of chain-end defects induces both stress relaxation and concentration into finite-sized regions of the crystallite. The failure response of both perfect and defective crystallites, and the analysis of observed failure mechanisms and their causes is contained in Section 5, while Section 6 addresses the role of chain-end defects in determining the crystallite modulus. Concluding remarks and a discussion of possible applications of the results, as well as avenues for future work are contained in Section 7.

\section{Methods}

\subsection{Force field selection}

The primary potential used in this work is the REAXFF force field [23-26]. REAxFF is a reactive bond-order force field capable of modeling the breakage and formation of covalent bonds dynamically during a simulation. It has been used extensively to study fracture events in atomistic solids [28-33], making it a good candidate for studying bond rupture events and the failure behavior of crystalline PPTA. The primary challenge with using REAXFF is choosing a suitable parameter set to model PPTA, as no parameterization has been developed specifically for aromatic polyamides. A recent MD study [34] used the parameterization of Strachan et al. [35] for modeling both amorphous and crystalline phases of PPTA. However, our early 
simulations showed that this parameter set was not able to replicate the basic PPTA crystal structure (see Figure 1) at room temperature. In this work, we instead employ the parameterization of Liu et al. [27], which was adapted from that of Strachan et $a l$. This parametrization corrects the London dispersion forces in order to replicate more accurately the cell volume of several crystalline polymers, a good indication that it is appropriate for modeling PPTA. Validation of this choice of parameter set is discussed in Section 3.

REAXFF is a complex and computationally expensive force field that facilitates covalent bond rupture. In simulations where modeling covalent bond rupture is unnecessary, the PCFF force field is used. PCFF is a Class II force field [36] parameterized from $a b$ initio theoretical calculations, and is designed specifically to model polymers and organic compounds. The PCFF force field has been used in MD studies of PPTA $[14,16,37,38]$ to calculate molecular geometry, possible chain conformations, physical properties like the crystalline elastic moduli and coefficient of thermal expansion, and buckling behavior under axially compressive loading. Grujicic et al. [17-22] use a more recently parameterized Class II force field known as COMPASS [39] to conduct a variety of MD simulations of PPTA, exploring, among others, the effects of various classifications of defects and the effect of different static loading conditions on the material response. The use of PCFF and its similar counterpart COMPASS in previous successful MD modeling efforts justifies its use here for modeling PPTA crystallites under loading conditions where primary bonds are not expected to rupture.

\subsection{Molecular model}

The simulations performed in this work were conducted using LAMMPS [40], a parallelized MD code capable of handling large-scale atomic simulations. Initial atomic models of crystalline PPTA were created in Materials Studio [41]. Perfect crystal models were created from an atomic topology file based on the X-ray diffraction measurements by Northolt [1]. A perfect crystal model of any size can be created from this starting point by replicating the crystalline unit cell $N_{x}$, $N_{y}$, and $N_{z}$ times in the $x, y$, and $z$ directions, respectively. The only type of structural crystallite defects considered in this work are chain-end defects. Chain- 
end defects are introduced by removing the amide linkage between two aromatic rings along the chain backbone and capping the aromatic rings with hydrogen atoms, as demonstrated in Figure 3. In true aramid fibers, chain-ends can be capped with a variety of different functional groups, as explored in [18]. However, in this work only the simple manifestation shown in Figure 3 is considered with the goal of focusing on the impact of introducing finite-length chains rather than exploring the influence of different types of chain-ends on overall crystallite properties.

All MD simulations performed here make use of periodic boundary conditions in order to simulate PPTA crystallites in a bulk environment. Defect-free crystallite models represent infinite length PPTA chains, since backbone chain bonds are continuous across periodic boundaries in the $z$-direction. The inclusion of chain-end defects results in models that represent finite-length chains. One model frequently used in this work is simulation cell of $N_{x} \times N_{y} \times N_{z}$ crystalline unit cells, with one chain-end defect introduced into each PPTA chain. This yields a simulation cell where each polymer chain is approximately $N_{z}$ monomers in length. By changing the length of the simulation cell $N_{z}$ and introducing defects at different locations, it is easy to create crystallite models of different polymer chain lengths and defect distribution patterns.

There are two fundamental types of MD simulations performed in this work. The first is the simulation of a PPTA crystallite under constant temperature and pressure conditions, with the goal of obtaining time-averaged properties of the crystallite at these fixed conditions. To regulate the temperature and pressure in these simulations, a Nosé-Hoover chain thermostat [42-44] was used. Thermostat/ barostat time constants of $100 \mathrm{fs}$ for temperature and $1000 \mathrm{fs}$ for pressure were used for both REAxFF and PCFF simulations. The second type of simulation imposes dynamic uniaxial stress loading on the PPTA crystallite in the fiber direction via constant (axial) strain-rate deformation. To conduct these simulations, the PPTA crystallite system is first equilibrated at a stress-free state to obtain the equilibrium crystallite dimensions $L_{x_{0}}, L_{y_{0}}$, and $L_{z_{0}}$. Then, one end of the simulation cell is held fixed, while the other is stretched at a constant velocity $v_{0}=\dot{\epsilon}_{z z} L_{z_{0}}$, where $\dot{\epsilon}_{z z}$ is the desired applied strain rate in the longitudinal direction of the chain. Decoupling the pressure components in the system equations of motion, as described in [44], allows velocity boundary conditions to be specified in the z-direction on the axial simulation 
cell faces while maintaining a zero time-averaged stress on the lateral cell faces. This results in dynamic uniaxial stress loading of the simulation cell via constant axial strain-rate deformation. The constant strain-rate simulation methodology used here is similar to that used by Hossain et al. [45] to simulate dynamic strain-rate loading of amorphous polyethylene.

The REAxFF and PCFF force fields have fundamentally different algorithmic implementations, and as such require different sets of simulation-specific settings. The REAXFF force field parameters are specified from the parameter file in the supplemental material in [27]. For REAxFF, a time step of $\Delta t=0.25$ fs is used to integrate the equations of motion. REAxFF uses the QEq charge equilibration method $[46,47]$ to compute and update atomic charges during the simulation, which is necessary to accommodate changes in covalent bonding topology. The tolerance for the QEq algorithm is set to $1.0 \times 10^{-6}$ coulomb, and the cutoff distance used for charged interactions is set to $10.0 \AA$. The implementation of the REAxFF algorithms in LAMMPS is described in [48].

The PCFF force field parameters are obtained from Materials Studio using the tool msi2lmp packaged with the LAMMPS software. The Lennard-Jones coefficients for interactions between atoms of type $i$ and $j$ are determined using a mixing rule as follows:

$$
\begin{aligned}
\epsilon_{i j} & =\frac{2 \sigma_{i}^{3} \sigma_{j}^{3} \sqrt{\epsilon_{i} \epsilon_{j}}}{\sigma_{i}^{6}+\sigma_{j}^{6}} \\
\sigma_{i j} & =\left(\frac{\sigma_{i}^{6}+\sigma_{j}^{6}}{2}\right)^{1 / 6}
\end{aligned}
$$

where $\epsilon_{i}$ and $\sigma_{i}$ are the Lennard-Jones parameters for atom $i$. The Lennard-Jones cutoff is set to $10.0 \AA$. Charge interactions in PCFF are computed using the PPPM method [49], with a relative force error tolerance of $1.0 \times 10^{-4}$ for the solver, and a long-range cutoff of $10.0 \AA$. A time step of $\Delta t=1.0 \mathrm{fs}$ is used to integrate the equations of motion of the system. 


\section{Force field validation}

\subsection{Unit cell geometry}

The ability to replicate basic molecule and crystalline unit cell geometry is an essential characteristic of any potential chosen to model PPTA. As such, the crystalline unit cell dimensions and molecular geometry of each force field under an unloaded, equilibrated state are computed and compared to literature and predictions from other MD studies. The X-ray diffraction measurements of Northolt [1] yield the crystalline unit cell dimensions as well as the atomic coordinates, bond lengths, and angles in the system. For the purpose of discussing relevant geometric features, Figure 4 provides a sketch of the monomer geometry and identifies 7 distinct bonds. These bond labels coincide with the 7 bond types in the PCFF force field parameterization.

For both PCFF and REAXFF, simulation cells consisting of $4 \times 4 \times 8$ unit cells (in the $x, y$, and $z$ directions, respectively) of perfectly crystalline PPTA were created. Periodic boundary conditions were used to represent bulk crystalline PPTA. The system was first equilibrated at a temperature of $300 \mathrm{~K}$ and zero pressure, representing unloaded PPTA at room temperature, for a total of 100 ps. After equilibration, geometric quantities of interest were time-averaged for $200 \mathrm{ps}$ in each force field. Quantities measured include the simulation cell dimensions, bond lengths and angles, and aromatic ring orientations as represented by their time-averaged plane normal direction cosines. These time-averaged quantities are reported in Table 1, alongside the values measured by Northolt's X-ray diffraction experiments. The bond numbers reference the labeling in Figure 4, and the aromatic ring and link numbering follows the diagram in Figure 5. As per convention, the hydrogen bond distance is given as the $\mathrm{O}-\mathrm{N}$ distance between adjacent chains, where the atoms $\mathrm{O}-$ $\mathrm{H}-\mathrm{N}$ form the hydrogen bond triplet. Angles 2-3 and 2-5 refer to the angles formed by Bonds 2 and 3, and by Bonds 2 and 5, respectively.

Agreement between experiment and each potential is reasonable. PCFF is able to more closely replicate the experimentally measured crystalline unit cell dimensions, while REAXFF overestimates $c$ and underestimates $b$. The smaller value of $b$ in REAXFF is consistent with the shorter hydrogen bond length computed with this force field, an indication that the hydrogen bonding forces are stronger in REAXFF 
PCFF ReaxFF Northolt [1]

\section{Unit cell dimensions $( \pm 0.01 \AA)$}

$a$
$b$

Bond length $( \pm 0.01 \AA)$

Bond 1 length

Bond 2 length

Bond 3 length

Bond 4 length

Bond 5 length

Bond 6 length

Bond 7 length

Hydrogen bond $(\mathrm{O}-\mathrm{N})$

Angles ( $\pm 0.1 \mathrm{deg})$

Angle 2-3

Angle 2-5

Ring plane normal direction cosines $( \pm 0.001)$

$\begin{array}{rrc}8.01 & 7.69 & 7.87 \\ 5.19 & 4.96 & 5.18 \\ 12.96 & 13.33 & 12.90\end{array}$

1.21

1.37

1.44

1.00

1.50

1.40

1.10

3.10

124

111

124

123

125

117

$$
\begin{aligned}
& \text { Ring } 1 \cos \theta_{x} \\
& \text { Ring } 1 \cos \theta_{y} \\
& \text { Ring } 1 \cos \theta_{z} \\
& \text { Ring } 2 \cos \theta_{x} \\
& \text { Ring } 2 \cos \theta_{y} \\
& \text { Ring } 2 \cos \theta_{z}
\end{aligned}
$$

Cross-ring direction cosines $( \pm 0.001)$

$$
\begin{aligned}
& \text { Ring } 1 \cos \theta_{z} \\
& \text { Ring } 2 \cos \theta_{z}
\end{aligned}
$$

$\begin{array}{lll}0.837 & 0.821 & 0.821 \\ 0.530 & 0.558 & 0.557 \\ 0.136 & 0.121 & 0.125 \\ 0.864 & 0.881 & 0.838 \\ 0.434 & 0.462 & 0.554 \\ 0.254 & 0.099 & 0.066\end{array}$

$\begin{array}{lll}0.957 & 0.975 & 0.968\end{array}$

$\begin{array}{lll}0.997 & 0.987 & 0.994\end{array}$

Table 1: Equilibrium molecular geometry computed with PCFF and ReaxFF, compared to the X-ray diffraction measurements by Northolt [1]. The uncertainty labels in the left column headings refer to the standard error in the time averages for the measurements taken here with PCFF and ReaxFF. 
compared to PCFF. All covalent bond lengths in each force field are in good agreement with experimental results, with the exception of Bond 2 in REAxFF, which is about $8 \%$ longer than the value in either PCFF or experiment. It is important to note that despite a few discrepancies in the details of molecule geometry relative to experimental data and simulation results using PCFF, REAxFF does predict the correct bonding topology for the molecule, which is a necessary condition for its use in modeling PPTA.

\subsection{Calculation of elastic moduli}

The ability of an interatomic potential to reproduce accurate elastic moduli for the material being modeled is a good indication that the choice of potential is appropriate. Due to the symmetry of the PPTA unit cell, it is expected that the elastic moduli will have orthotropic symmetry, requiring nine independent constants to describe the elasticity of the material. The (instantaneous) stress is computed from the standard MD virial formula [50],

$$
\sigma_{i j}^{M D}=\frac{1}{V} \sum_{\alpha=1}^{N}\left[-m^{\alpha} v_{i}^{\alpha} v_{j}^{\alpha}+\sum_{\beta>\alpha}^{N}\left(r_{i}^{\beta}-r_{i}^{\alpha}\right) f_{j}^{\alpha \beta}\right]
$$

where indices $\alpha$ and $\beta$ refer to atoms and indices $i$ and $j$ refer to vector components, such that $m^{\alpha}$ is the mass of atom $\alpha, v_{i}^{\alpha}$ is the $i$-th velocity component of atom $\alpha$, $r_{i}^{\alpha}$ is the $i$-th position component of atom $\alpha, f_{j}^{\alpha \beta}$ is the $j$-th force component on atom $\alpha$ by atom $\beta, V$ is the volume of the atomic system, and $N$ is the number of particles in the system. The continuum mechanical stress can then be computed as the time-average of the MD stress, that is,

$$
\sigma_{i j}=\left\langle\sigma_{i j}^{M D}\right\rangle
$$

For linear elasticity, the stress-strain relation is given by

$$
\sigma_{i j}=C_{i j k l} \epsilon_{k l}
$$

where $\sigma_{i j}, \epsilon_{i j}$ represent the components of the stress and infinitesimal strain, respectively, while $C_{i j k l}$ are the components of the fourth-order elasticity tensor, and repeated indices imply summation. Due to the symmetry of stress and strain, 
this relation can be written conveniently in matrix form as

$$
[\boldsymbol{\sigma}]=[\mathbf{C}][\boldsymbol{\epsilon}]
$$

where $[\boldsymbol{\sigma}]=\left[\begin{array}{lllll}\sigma_{x x} & \sigma_{y y} & \sigma_{z z} & \sigma_{x y} & \sigma_{y z} \sigma_{x z}\end{array}\right]^{T}$ and $[\boldsymbol{\epsilon}]=\left[\epsilon_{x x} \epsilon_{y y} \epsilon_{z z} 2 \epsilon_{x y} 2 \epsilon_{y z} 2 \epsilon_{x z}\right]^{T}$, and $[\mathbf{C}]$ is a symmetric $6 \times 6$ matrix.

To calculate the full set of elastic moduli for crystalline PPTA at room temperature (taken to be $300 \mathrm{~K}$ ), six independent simulations are conducted, each of which applies one of the six fundamental deformation modes (three normal and three shear) to the simulation cell. A simulation cell of $3 \times 5 \times 2$ PPTA crystalline unit cells is used, which yields roughly a cube-shaped simulation cell. The stress is time-averaged while the simulation cell is held in the deformed configuration, and each simulation yields six elastic constants. For each simulation, a strain of $\epsilon=0.004$ is used (in the case of shear strain, this would correspond to, for example, $\left.2 \epsilon_{x y}=\epsilon=0.004\right)$, which was deemed small enough to fall within the infinitesimal strain approximation of linear elasticity theory, yet large enough that the fluctuations in stress deviated sufficiently from zero as to give meaningful time-averaged values. Perfect symmetry of $[\mathbf{C}]$ is not reproduced due to the natural variance which occurs in finite-length time-averages of the stress components; similarly, some entries in [C] which are expected to be zero due to orthotropic material symmetry deviate slightly from zero. Since these features are attributed to the aforementioned natural errors in the time-averaging of the stress components, the actual reported values of [C] are taken by first zeroing all of the components which vanish for a standard orthotropic compliance matrix, and taking $[\mathbf{C}]$ to be the symmetric part of its computed approximation.

The results of the moduli calculations are presented in Table 2, alongside the results from two previous MD studies of the elastic moduli of crystalline PPTA, one using the PCFF force field to compute the moduli at a temperature of $300 \mathrm{~K}$ [14], and the other using DREIDING to compute the moduli at $0 \mathrm{~K}$ [51]. The discrepancies in elastic moduli values for PCFF calculated here versus the PCFF calculations by Lacks and Rutledge [14] can be attributed to differences in methodology. Lacks and Rutledge calculate the moduli by numerically estimating the temperature-dependent Helmholtz free energy in a static crystallite system, while the method here computes the time-average of the stress in system vibrating dynamically at finite temperature. 
Despite the difference in methodology, reasonable agreement is obtained.

There are some significant differences between values computed here using PCFF and REAxFF, most notably in the $C_{22}$ and $C_{33}$ values, which are associated with the normal stiffnesses $E_{y y}$ and $E_{z z}$, respectively. Comparing $E_{y y}$ and $E_{z z}$ values in REAXFF with those from DREIDING shows comparable results, suggesting that the REAXFF predictions are reasonable. It should be noted that the DREIDING values were computed at a temperature of $0 \mathrm{~K}$, and should therefore be stiffer compared to DREIDING predictions at $300 \mathrm{~K}$ - this would yield a more favorable comparison with $E_{z z}$ in REAxFF, but a less favorable one with $E_{y y}$. The higher values of $E_{y y}$ and $E_{z z}$ in REAxFF compared to PCFF can be attributed to stronger hydrogen bonding (due to shorter hydrogen bond length as discussed in Section 3.1) and stiffer backbone chain bonds, respectively.

Experimental measurements for the crystalline elastic modulus $E_{z z}$ have yielded estimates in the 200-260 GPa range [52-54], while theoretical estimates have placed the same modulus in the 180-200 GPa range [13, 55]. It appears that all MD potentials list in Table 2 yield an overestimate of $E_{z z}$ compared to theory and experiment. It is worth noting that both PCFF and REAxFF are parametrized using data from quantum mechanical calculations, and such parametrizations are known to overestimate elastic moduli [56]. All of these points considered, the computed moduli here are deemed accurate enough to consider both PCFF and REAXFF capable of reasonably representing the elasticity properties of crystalline PPTA.

\subsection{Molecular geometry changes with axial stretch}

Much of the work presented here concerns simulating crystalline PPTA at elevated levels of axial strain. Since the authors are not aware of any experimental or theoretical data predicting how PPTA chains deform under tensile loading, simulations are now conducted to examine the change in molecular geometry with crystallite strain. The predictions made by REAXFF with respect to changes in bond lengths, angles, etc., with crystallite strain are compared against these same predictions made by PCFF. Individual simulations are performed at crystallite strains between $0 \%$ and $7 \%$, at increments of $1 \%$. Geometric features, such as 


\begin{tabular}{ccccc}
\hline Component & $\begin{array}{c}\text { PCFF } \\
(\mathbf{3 0 0} \mathbf{K})\end{array}$ & $\begin{array}{c}\text { ReaxFF } \\
(\mathbf{3 0 0} \mathbf{K})\end{array}$ & $\begin{array}{c}\text { Lit. PCFF [14] } \\
(\mathbf{3 0 0} \mathbf{K})\end{array}$ & $\begin{array}{c}\text { Lit. DREIDING [51] } \\
(\mathbf{0} \mathbf{K})\end{array}$ \\
\hline$C_{11}$ & 11.9 & 15.9 & 11.9 & 15.3 \\
$C_{22}$ & 23.6 & 91.9 & 32.2 & 82.3 \\
$C_{33}$ & 293 & 324 & 284 & 338 \\
$C_{44}$ & 3.9 & 2.7 & 6.1 & 5.2 \\
$C_{55}$ & 9.1 & 8.4 & 14.3 & 15.4 \\
$C_{66}$ & 0.2 & 0.9 & 0.5 & 0.7 \\
$C_{12}$ & 11.3 & 15.6 & 11.1 & 5.6 \\
$C_{23}$ & 7.0 & 32 & 14.2 & 9.6 \\
$C_{31}$ & 9.3 & 10 & 10.5 & 4.4 \\
\hline$E_{x x}$ & 10.5 & 15.2 & 10.9 & 80.1 \\
$E_{y y}$ & 20.9 & 87.5 & 29.5 & 336 \\
$E_{z z}$ & 291 & 320 & 281 & \\
\hline
\end{tabular}

Table 2: Elastic constants (units in GPa) computed here using the PCFF and ReaxFF force fields, alongside literature calculations. All values are computed at a temperature of $300 \mathrm{~K}$, with the exception of the DREIDING force field values, which were computed at $0 \mathrm{~K}$. The standard error in the time averaging of the elastic constants performed here is $\pm 1 \mathrm{GPa}$. 
molecular bonds, angles, etc., are computed via time averaging at each strain increment, and emphasis is placed on observing the mechanisms by which the PPTA chains accommodate stretching in each force field.

The change in molecular bond length, strain, and orientation as a function of monomer strain is plotted in Figures 6-8. One important conclusion from the results is that Bond 2 is not subjected to significant stretching relative to the overall monomer stretch. This is particularly true for REAxFF, where Figure $7 \mathrm{~b}$ highlights that Bond 2 essentially undergoes no change in length as the monomer is stretched to a strain of $7 \%$. These observations can be explained by examining Figure 8, which shows the change in the direction $\operatorname{cosine} \cos \theta_{z}$ in each bond versus monomer strain, where $\theta_{z}$ represents the bond's orientation relative to the fiber $z$ axis. It can be seen that Bonds 3 and 5 begin highly aligned with the $z$ axis and remain so as monomer strain is increased, but $\cos \theta_{z}$ for Bond 2 increases with monomer strain, indicating that it becomes more closely aligned with the $z$ axis as the monomer is stretched. As is evident from the monomer diagram in Figure 4, a rotation of Bond 2 to align with the $z$ axis would increase the length of the monomer, though it need not induce a commensurate increase in the length of Bond 2. Even though Bonds 3 and 5 stretch significantly more in PCFF than in REAxFF, each force field behaves comparably in that they predict accommodation of a substantial portion of the monomer stretch through rotation (rather than stretching) of Bond 2.

In addition to examining individual bonds, it is also of interest to consider the contributions of larger parts of the polymer chain to the monomer stretch. To study this, a single monomer is broken into four length segments as depicted in Figure 5. The segments consist of the span of the two aromatic rings, as well as the span of the two "links" which connect these rings. Together, these four segments span the length of a single monomer, and the strain contributions of each as the monomer is stretched are plotted in Figure 9. From this figure, it is clear that the two potentials accommodate stretching of the monomer in slightly different ways. In PCFF, Links 1 and 2 as well as Ring 1 make similar contributions to the total monomer strain, while Ring 2 stretches significantly less. In REAxFF, the majority of the stretch is accommodated nearly equally by Rings 1 and 2, while Links 1 and 2 make a smaller contribution than in PCFF. Also, while not plotted here, the additional strain accommodated by the aromatic rings in REAXFF is due to the bending of 
the angles within each ring, which allows the span of the rings to increase without significantly increasing the length of the bonds within the rings.

These results show both similarities and differences in the deformation of a PPTA chain when modeled with PCFF and REAXFF. Both force fields predict that Bond 2 rotates rather than stretches to accommodate the monomer stretch. The major difference between the force fields is in how bond angles change, with REAXFF exhibiting more flexible angle changes as evidenced by Bond 2 essentially not changing its length, and by the aromatic ring span increasing more significantly than in PCFF. This is likely because in REAxFF the forces contributed by the angle terms tend towards zero as the bond order decreases; PCFF, on the other hand, models the angles as nonlinear springs with finite stiffness at any level of deformation. Ultimately, the predictions made by REAxFF are similar enough to those made by PCFF to deem it appropriate for modeling the deformation of PPTA at high strains.

\section{Zone of influence of chain-end defects in the PPTA crystallite}

\subsection{Influence of a single isolated defect}

To better understand how chain-end defects affect the behavior of PPTA crystallites, the impact of a single isolated defect on the local behavior of nearby chains is studied. It is expected that a defect will cause some load transfer from the broken chain to adjacent surrounding chains, resulting in stress relaxation in the broken chain and stress concentration in adjacent chains near the defect site. The region in which chains deviate from nominal behavior will be of finite size, and the volume covered by this region will be referred to as the "zone of influence" of a single defect. The goal of the study is to determine the size of this zone of influence, and how it affects the behavior of neighboring chains.

To perform the study, a model of $4 \times 8 \times 32$ crystalline PPTA unit cells is used. This relatively large system size was chosen to ensure that it was much larger than the zone of influence of the defect to be introduced. A single chain break is introduced into the center of this system, while all other chains remain intact, and of effective 
infinite length due to the use of periodic boundary conditions. A fixed strain $\epsilon_{z z}$ is applied to the system while a constant temperature of $300 \mathrm{~K}$ and zero timeaveraged pressure on the lateral sides of the simulation cell are maintained by an NPT barostat/thermostat. Under these conditions, the geometric configuration is averaged in time, and the resulting structural features are analyzed. In particular, the strain of each link in the chain (as diagrammed in Figure 5) is used to detect stress concentrations and relaxations. In order to determine if the zone of influence changes with applied strain, this study is performed on systems strained to $2 \%, 4 \%$, and $6 \%$, and both the PCFF and REAxFF force fields are examined to see how they behave relative to one another.

The results of the simulations are summarized in Figure 10. It is found that only the broken chain and two nearest adjacent chains are influenced by the chain-end defect, with all other links in the system remaining at the nominal levels of link strain in a perfect crystal. For this reason, only the response of the three chains influenced by the defect are displayed in Figure 10. The figure plots the strain in each link of the chain relative to the nominal strain of a link in a perfect crystal at the indicated level of crystallite strain. Both the PCFF and REAxFF force fields predict very similar chain behavior. The broken chain experiences a region of relaxation along the chain, roughly \pm 8 monomers away from the defect site in both PCFF and REAxFF, after which the link strain returns to the nominal value in a perfect crystal. This means that the hydrogen bonds provide an efficient shear-like transfer mechanism such that the broken chain carries the same load as the intact chains in monomers at least 8 monomers away from the chain-end. In the adjacent chains, there is an increase in the link strain near the defect, with the affected region spanning approximately \pm 4 monomers for both force fields. The response of the two adjacent chains is asymmetric relative to one another, which can be explained by observing the details of the crystallite geometry near the chain-end defect as depicted in Figure 3. In the chain above the defect, the oxygen atom extending from the link is the atom closest to the defect, whereas in the chain below the defect, the hydrogen atom extending from the link is nearest the defect. Hence, there is a geometrical asymmetry that is responsible for the difference in the response in the two adjacent chains. The raised levels of link strain in these adjacent chains is indicative of stress concentration and increased load-bearing to offset the decreased load-bearing capacity of the broken 
chain near the defect site.

In addition to the change in link strain, the change in hydrogen bond stretch near the defect site is also examined, and the results are displayed in Figure 11. It is found that only hydrogen bonds attached to the broken chain experienced detectable deviations from the nominal hydrogen bond stretch in the crystal. For this reason, there are two rows of bonds whose response is displayed, which are bonds between the broken chain and the chains located \pm 1 -monomer adjacent to the broken chain. Hydrogen bonds take on significant additional stretch only at the highest strain examined here $(6 \%)$. The region of additional stretch spans approximately \pm 4 monomers along the length of the chain. Figure 12 offers visualizations of the molecular deformation near the defect site at strains of $2 \%$ and $6 \%$. It is clear from this figure that the increased hydrogen bond stretch near the defect site is due to the relaxation of the broken chain near that site. The chain relaxation causes the broken chain to decrease in length relative to the other intact chains in the system, and hydrogen bonds near the chain-end slip relative to adjacent chains in response to this. This slippage is exacerbated at higher crystallite strain, hence the significant difference in hydrogen bond stretch between the cases of $2 \%$ and $6 \%$ strain. In bonds \pm 4 monomers away from the chain end, the chain stretch in the broken chain begins to approach its nominal value (as evidenced in Figure 10), and hydrogen bond slippage is mitigated.

From this analysis on the impact of defects on nearby hydrogen bond stretch, it may appear that the zone of influence of the defect increases in size with crystallite strain. It is important, however, to realize that hydrogen bonds only take on additional stretch when chain slippage occurs near the defect site. Since links in the broken chain returns to nominal levels of stretch 8 monomers away from the defect, hydrogen bond slippage will not occur further away from the defect than this. While the size of the zone of influence is confirmed constant only up to strains of $6 \%$ here, there are no indications that suggest it would change at higher strains provided it did not induce primary bond rupture. Hence, it appears that the zone of influence of a single defect is restricted to a region spanning \pm 8 monomers along the broken chain, and spanning \pm 4 monomers along the two chains immediately adjacent to the chain-end defect site. 


\subsection{Influence of multiple defects}

With the role of isolated defects on molecular deformation having been examined, the impact of multiple interacting defects (that is, defects lying within each other's zones of influence) is now studied using three examples, all of which introduce a defect pattern into a single hydrogen-bonded sheet of $4 \times 8 \times 32$ crystalline unit cells. In the first example, defects are introduced at the -4 and +4 monomer positions, segmenting the broken chain into two chains of 8 and 24 monomers in length, the shorter of which lies within the zone of influence of both defects. The resulting chain behavior is plotted in Figure 13. As with the case of the single chain-end defect displayed in Figure 10, the only chains whose behavior is affected by the defects are the broken chain itself and the two in-sheet chains immediately adjacent to the broken chain. Between the -4 and +4 monomer positions, links in the short broken chain do not have an opportunity to return to the nominal link strain. A return to nominal link strain can be seen to occur at about the \pm 12 monomer positions, indicating that the size of the relaxed region along the larger broken chain is within 8 monomers of the two defects, which is consistent with the size of the relaxed region near defects observed in Figure 10. In the adjacent chains, the increase in link strain closely follows the behavior observed for the single chain-end case in Figure 10. Therefore, the presence of two interacting defects does not seem to exacerbate the stretching or relaxation of the links in nearby chains or enlarge the zone of influence of either defect. The hydrogen bond behavior (not plotted here) is also essentially identical to that displayed in Figure 11, suggesting that interacting defects do not weaken nearby hydrogen bonds any more than if these defects were isolated from one another.

The defect pattern used for the second example is displayed in Figure 15. In this case, three linkages in a row are eliminated, creating a larger gap between chain-ends. Figure 15 shows the results for this case for both the PCFF and REAXFF force fields. It is observed that there is no substantial difference in behavior between this case and the case of a single missing link (Figure 10). Indeed, the zone of influence of the defect is approximately the same, and the extent of the chain relaxation/stretching compared to the single missing link case is also approximately the same. Once again, the affected chains are constrained to the broken chain and 
the two immediately adjacent chains in the same hydrogen-bonded sheet. Also, the hydrogen bond response is essentially identical to that displayed in Figure 11.

The third and final example is that of a "crack", where three chain-ends are introduced at the same location in three consecutive adjacent chains, as illustrated in Figure 16a. In this example, the observed chain behavior was substantially different than that in the other cases considered here. The zone of influence expanded beyond \pm 8 monomers relative to the broken chains at strains higher than $4 \%$, and also expanded to include to two unbroken chains relative to the broken chains at the edges of the crack. These results can be seen by examining the plots of the chain responses in Figure 17. Hence, defects which lie close to each other in adjacent chains have a much stronger effect on chain behavior than when defects are stacked closely to each other along a single chain, as they were in previous examples here. The stark difference in behavior between these cases occurs due to hydrogen bond breakage and subsequent chain slippage that occurs for chains near the crack tip, which is visualized in Figure 16b. The extent of the slippage is much less severe at lower crystallite strains where the zone of influence is restricted to a smaller portion of the broken chain, which explains why the zone of influence increases with increasing strain. It is interesting to note that the chain-slippage experienced at the crack tip is essentially a crack tip blunting mechanism such as that observed in fiber-reinforced composites and ceramics [57]. The slippage of the chains at the crack tip reduces the peak stretch experienced by the adjacent intact chains near the crack tip, and therefore mitigates in-plane crack extension via a crack-like deflection mechanism.

The major conclusions drawn from examining the impact of interacting defects is that when chain-ends are placed near each other in an end-to-end configuration, such as the case of the results displayed in Figure 13, the stress relaxation/concentration due to the interacting defects appears to be approximately additive in their overlapping regions, and there is no exacerbation of stress concentrations or relaxations relative to the case when defects are spread further apart. This also holds true for the case of larger gaps between chain-ends, such as for the configuration depicted in Figure 14. However, when chain-end defects are placed near each other in adjacent chains, such as the configuration in Figure 16, the disruption of the crystal structure is much more severe and widespread, leading to easier slippage of hydrogen bonding between chains, particularly at crystallite strains of $4 \%$ and higher. These 
observations give key insights into how defects influence the overall integrity of the crystallite structure.

\section{Failure of PPTA crystallites under constant strain-rate loading}

\subsection{Failure of defect-free crystallites}

Attention is now turned to exploring the mechanical response of PPTA crystallites under dynamic strain-rate loading, with particular focus given to the stress-strain response and mechanisms involved in the failure of the PPTA crystallite. To study the failure of a perfect crystal, a simulation is conducted where a perfectly crystalline array of $4 \times 4 \times 8$ PPTA unit cells is subjected to constant strain-rate deformation at a rate of $\dot{\epsilon}_{z z}=1.0 \times 10^{9} \mathrm{~s}^{-1}$. The REAXFF force field is used to allow for the breakage of primary bonds along the PPTA chain backbones. The results of this simulation are displayed in Figure 18, where the stress $\sigma_{z z}$ (calculated according to equation (2)) is plotted versus engineering strain $\epsilon_{z z}$ for the system, together with the number of primary bond ruptures (bonds of types 2, 3, and 5 along the backbone of the polymer chain, as diagrammed in Figure 4) and secondary hydrogen bond ruptures. REAXFF tracks only bond order between atoms rather that the formal existence of a bond, but a low bond order is a good indicator that a bond has ruptured. For example, Chenoweth et al. [58] indicate bond failure when the bond order falls below 0.2. Here, a bond order of 0.3 was found to be a good indicator of failure of the primary backbone bonds based on visualizations of bond failure in strain-to-failure simulations. Following methods from the literature [59, 60], hydrogen bonds are taken to exist if the bond length $d_{h b}$ and the bond angle $\theta_{h b}$ satisfy the conditions $d_{h b}<3.5 \AA$ and $\theta_{h b}<120^{\circ}$. Here, the bond length is defined as the distance between $\mathrm{O}$ and $\mathrm{N}$ atoms in the bond, while the bond angle is defined as the angle formed between $\mathrm{O}-\mathrm{H}-\mathrm{N}$ atoms (see Figure 19).

There are several important conclusions drawn from the results in Figure 18. Starting with the stress-strain response, careful observation reveals that the stressstrain curve undergoes a slight non-linearity after exceeding about $0.6-0.7 \%$ strain, transitioning from an initial modulus $E_{z z}=320 \mathrm{GPa}$ to $355 \mathrm{GPa}$. After this initial 
increase, the modulus remains constant up until primary bonds begin to break, at which point the stress begins to drop. Hence, the stress response of the crystallite is nearly linearly elastic before the onset of failure.

In the strain-to-failure simulations performed here, system failure is defined as the point at which the maximum stress is attained, and the strain commensurate with this stress level is called the failure strain, and is denoted $\epsilon_{f}$. Note that the tensile strength observed here, estimated at approximately $35 \mathrm{GPa}$ in Figure 18, is about an order of magnitude higher than typical experimentally measured strengths in Kevlar fibers [61]. This is not surprising given the complex microstructure found in true Kevlar fibers compared to the defect-free and perfectly aligned nature of the chains in the crystallites simulated here. After the onset of failure, the stress response undergoes a "stair-step" pattern, until it reaches a zero stress value, indicating complete failure of the system. By examining the plot of bond breaks versus strain, it can be seen that the rises in stress in the post-failure regime correspond to portions of the loading in which no primary backbone bonds rupture; hydrogen bonds also appear to heal and re-engage during these periods. This phenomenon can be better explained by examining Figure 20, which displays the same stress-strain plot as Figure 18, but substitutes the bond break plot with a plot of broken backbone bonds organized by hydrogen-bonded sheet. There are two monomers per unit cell, so in the $4 \times 4 \times 8$ unit cell system there are a total of 8 sheets present in the simulation cell. Upon examining Figure 20, it is clear that each of the three stress drops in the post-failure regime correspond to a group of sheets failing at roughly the same time: the first group of failures consists of Sheets 2, 6, and 8, the second group of Sheets 3, 4, and 5, and the third group of sheets 7 and 1. After all sheets have failed, the system stress drops to zero, and the system can no longer support any load. Furthermore, the first bond to rupture within each sheet is of Type 2. Thus, bonds of Type 2 are identified as the weakest bond along the polymer chain backbone, and therefore the limiting factor in the overall strength of the crystallite. Identification of the weakest backbone PPTA chain bond is an important insight in understanding the factors governing the failure strength of individual crystallites.

To determine whether the observed crystallite behavior is consistent across different initial simulation conditions and loading rates, a set of five simulations at a loading rate of $\dot{\epsilon}_{z z}=1.0 \times 10^{9} \mathrm{~s}^{-1}$ are performed, along with one simulation each 
at loading rates of $\dot{\epsilon}_{z z}=2.0 \times 10^{8} \mathrm{~s}^{-1}$ and $\dot{\epsilon}_{z z}=4.0 \times 10^{7} \mathrm{~s}^{-1}$. The stress-strain curves for the five simulations at a strain rate of $1.0 \times 10^{9} \mathrm{~s}^{-1}$ are presented in Figure 21, and the stress-strain and bond break curves for simulations performed at strain rates of $2.0 \times 10^{8} \mathrm{~s}^{-1}$ and $4.0 \times 10^{7} \mathrm{~s}^{-1}$ are presented in Figure 22. In all cases, Type 2 bonds are the first to break in each sheet, demonstrating that this behavior is not dependent on initial conditions or the rate of loading of the crystallite. Examining Figure 21 shows that the onset of bond failure depends on initial conditions, but Type 2 bonds are the first to fail in all five runs (not plotted here), indieating that Type 2 bonds are the weakest in the polymer chain regardless of crystallite initial eonditions. Figure 22 shows that Type 2 bonds are the first to fail regardless of applied strain-rate. The erystallite modulus and bond failure mechanisms do not appear to have any dependence on strain-rate, . That the perfect crystallite failure mechanism (Type 2 bond rupture) is independent of strain-rate is consistent with the nature of REAXFF, which does not include equations of motion for electrons and essentially represents instantaneously equilibrated electron distributions with changes in atomic position. Additionally, observing consistency of failure mechanism across these rates confirms that artifacts such as inertial effects, which may originate from such high rates of loading, are not present. It is therefore possible to proceed with analyzing failure mechanisms in crystallites containing defects without exploring the influence of strain-rate. Nevertheless, but a full exploration of the influence of strain-rate on the crystallite mechanical response is warranted given the use of aramid fibers in protective fabric subjecting to high rates of loading upon ballistic impact. This topic will be addressed in more depth in a subsequent article.

\subsection{Failure in crystallites containing chain-end defects}

With the failure of a perfect crystal analyzed, attention is now turned to failure in crystals containing chain-end defects. To begin, a system of $8 \times 8 \times 64$ unit cells, comprising chains of 64 monomers in length, is considered. Three variants of this system are studied. Variant I has its chain-ends distributed randomly throughout the simulation cell. In Variant II, all of the chain-ends are clustered in a region \pm 10 monomers about a plane bisecting the center of the simulation cell, whose normal vector is parallel to the $z$-axis. Variant III is similar to Variant II, but the clustering 
is constrained to an even smaller region of \pm 4 monomers about the bisecting plane. Figure 23 offers a schematic diagram of the chain-end locations for each variant. These distribution patterns are chosen to represent the different descriptions of chain-end distributions observed experimentally, as detailed in Section 1. Variant I is representative of the skin region of fibers, where chain-ends are thought to be distributed randomly, while Variants II and III are more representative of the fiber core, where chain ends are thought to be concentrated in periodic defect bands.

Figure 24 shows the stress-strain response of Variants I, II, and III, as well as that of the perfectly crystalline response, when modeled using REAXFF at a strain rate of $1.0 \times 10^{9} \mathrm{~s}^{-1}$. From these plots, it is clear that the biggest difference between the responses is the failure strain of the system. Among the systems including defects, Variant I is the strongest and Variant III is the weakest. This is because Variant III exhibits a weak plane with a high concentration of chain-end defects, which, per the analysis in Section 4, results in a region of weakened covalent and hydrogen bonds. Failure of the system is therefore governed by bond failure in this weak region. The strengths of these variants are lower than the simulated perfect crystallite strength of about $35 \mathrm{GPa}$, particularly for Variant III, whose strength of about $6 \mathrm{GPa}$ (as observed in Figure 24) is more closely aligned with the typically observed strengths of 2-4 GPa in Kevlar ${ }^{\circledR}$ fibers [61]. This suggests that Variant III is perhaps most representative among Variants I-III of the defect patterns found in actual Kevlar fibers, although there are many other factors at work (chain orientation, different types of defects not considered here, etc.) which contribute to the observed strength of these fibers. OneAn additional interesting point to extract from these results is that the modulus of Variants I-III is nearly the same, suggesting that only the concentration, rather than the distribution, of defects is what affects the modulus of the crystallites; further discussion on how chain-end defects influence crystallite modulus is included in Section 6.

Figure 25 displays the primary and secondary bond failure that occurs in each of Variants I, II, and III. These figures demonstrate that Variant I fails mostly due to primary bond ruptures. Variant II, where defects are clustered more closely together, fails due to a mixture of primary and secondary bond ruptures. Finally, Variant III fails due to almost exclusively secondary bond ruptures. Examining these results thus suggests that when defects are clustered closely together, the crystallite failure 
is governed by the strength of the secondary bonds in the crystal. When they are spread out, the strength of the crystallite is instead governed by the strength of the primary bonds. It is interesting to note that a related MD study [62] on chain-end defects in ultra-high-molecular-weight polyethylene (UHMWPE) fibers was carried out using the AIREBO reactive potential, where it was found that failure in systems containing chain ends always occurred due to chain slip rather than primary bond rupture. The presence of hydrogen bonds between adjacent chains in crystalline PPTA (which are absent in UHMWPE) is responsible for the difference in failure behavior between these types of polymer fibers.

To further explore why failure via hydrogen bonds occurs in favor of failure via primary bonds for the case of clustered defects, a crystallite model where chainend defect locations are deliberately chosen (rather than randomly assigned as in Variants I-III) is considered. Figure 26 displays a defect pattern, in which individual defects are spaced $\pm N$ monomers away from the bisecting plane of the simulation cell and in a zig-zag pattern, and are therefore spaced $2 N$ monomers apart from each other along the $z$-direction. The variable $N$ defines the size of the defect band in this system, and varying $N$ generates a set of patterns which are used to systematically explore the influence of chain-end defects on crystallite failure mechanisms. The pattern is implemented in a simulation cell of size $3 \times 8 \times 64$ unit cells, with the 64 monomer unit cell length intended to ensure the portion of the chains outside the defect band are mostly uninfluenced by the presence of the defects.

Strain-to-failure simulations using the REAxFF force field were conducted using defect band sizes of $N=1,2, \ldots, 8$ and a strain rate $\dot{\epsilon}_{z z}=1.0 \times 10^{9} \mathrm{~s}^{-1}$. It was observed that the crystallite failed via primary bond rupture for $N \geq 5$ and via secondary bond rupture and chain sliding for $N \leq 3$. Visualizations of sheet failure for the cases of $N=5$ and $N=3$ are shown in Figures 27 and 28, respectively. For the case $N=4$, it was observed that sheets could fail either by primary or secondary bond rupture. This failure behavior is consistent with the observations of the effects of chain-end defects on local bond behavior observed in Section 4. Recall that it was shown there that the zone of influence of a single defect was about \pm 8 monomers away from the defect site along the broken chain. For $N \geq 5$, defects are located about 10 monomers away from each other (due to the defect band size of $2 \mathrm{~N}$ monomers). Therefore, defects are too far away to interact with one another, and 
primary bonds break in adjacent chains near the defect site due to the increased load they bear. For $N \leq 3$, chain-end defects lie within each other's zones of influence, and hydrogen bonds become increasingly weak with decreasing $N$ due to the influence of the surrounding defects. Therefore, failure by chain-slippage and hydrogen bond rupture is the dominant mechanism. For the case $N=4$, bonds are on the edge of the zone of influence of the chain-end defects, such that either primary bonds or secondary bonds may fail. Because bond failure is stochastic in nature, the failure mode which occurs in each case depends on the relative probability of failure of each of the bond types in the system. For the case $N=4$, the fact that failure of primary or secondary bonds are both readily observed in the simulations implies that the probabilities of failure through each of these modes are of comparable magnitude, such that neither outcome is significantly more likely to occur than the other.

\section{Role of defects in crystallite modulus}

The presence of chain-end defects in the crystallite is expected to have a significant influence on the modulus of the crystallite. To investigate this conjecture, simulations were conducted where, starting from a perfect crystal cell of $N_{z}$ monomers in length, a single defect was introduced at a random location in each chain of the cell, yielding a crystallite consisting of chains $N_{z}$ monomers in length with the chain-end locations distributed randomly. The random distribution of chain-end defects is the only distribution considered for this study, which is motivated by the observation that the modulus of crystallite Variants I-III studied in Section 5.2 was unchanged despite the vastly different defect location distributions among the variants. Chain lengths of $N_{z}=4,6,8,12,16,24,32,48,64,96$, and 128 monomers were considered, and the resulting values for the modulus $E_{z z}$ of each system for both the PCFF and REAxFF force fields are plotted in Figure 29. The figure shows that increasing the chain length has a very strong effect on the increase in modulus initially, but this effect plateaus once a chain length of about 64 monomers is reached. This is an interesting insight, since chain lengths in typical aramid fibers are on the order of 150 monomers long. Since stiffness gains are minimal for chain lengths greater than 64 monomers, this suggests that a fiber manufacturer interested in improving fiber stiffness should look to improve on design parameters other than chain length (e.g., 
chain alignment and orientation relative to the fiber axis). However, long chains are still advantageous: per the discussions in Section 5.2, longer chains may have the benefit of minimizing defect concentrations, thereby increasing the overall strength of a fiber's underlying crystallite structure.

In order to better understand how the crystallite modulus depends on chain length, an analysis was conducted to determine which parts of the atomic structure contribute to the overall stiffness of the crystallite. For example, it is useful to know how much of the crystallite stiffness can be attributed to the stiffness of backbone chain bonds compared to the hindrance of crystallite stretching due to neighboring hydrogen bonds. To analyze this, certain terms in the interatomic potential were turned off, and this modified force field was used to compute the stiffness of the crystallite using the original MD trajectory generated from the unmodified version of the force field. In this way, the difference in stiffness which results from identical atomic motions, but different atomic force interactions, could be tracked. One analysis was conducted where all primary bond interaction terms were omitted from the force field, such that only pairwise interactions contributed to the stiffness of the system, ignoring the contribution of backbone chain stiffness. A second analysis was performed with $\mathrm{O}-\mathrm{H}$ hydrogen bond interactions omitted, essentially eliminating hydrogen bonds from contributing to the stiffness. Remarkably, the analyses showed that the modulus for the case of omitting hydrogen bond interactions was nearly unchanged compared to the true modulus, while the modulus was computed to be nearly zero when primary backbone bond contributions were omitted. These results were observed for both the PCFF and REAxFF potentials, offering strong evidence that the load in the crystal is borne almost entirely by the PPTA chain backbones, rather than by the surrounding secondary bonds. The surrounding bonds serve to keep the chains bound together in the lateral direction, essentially allowing the chains to act as a collection of parallel springs which determine the stiffness of the crystal.

Based on these observations, a model is proposed to predict the crystallite stiffness based on chain length (or equivalently, defect concentration) and uses a rule of mixtures approach. The model (whose full derivation is provided in Appendix A) assumes that the crystallite is a collection of chains of length $L_{c}$, which are long enough such that the chain-end defects are spread far enough apart as to be noninteracting. Each chain contains a region of length $L_{1}$ of undegraded stiffness (due to 
being far away from defects) and a region of length $L_{2}$ of degraded stiffness (due to being near defects), with associated crystallite stiffnesses $E_{1}$ and $E_{2}$ for each region. These assumptions lead to an expression for the defective crystallite stiffness $E$ as a function of chain length $L_{c}$ as

$$
\frac{E}{E_{1}}=\frac{1}{1+\frac{\phi}{L_{c}}},
$$

where $\phi=\left(\frac{E_{1}}{E_{2}}-1\right) L_{2}$ and can be treated as a material constant. To examine whether the model is suitable, a least-squares fit is performed on the PCFF and REAXFF modulus versus chain length data to estimate the constant $\phi$. The model assumes that chain-ends are placed far enough from one another such that there is no interaction between defects, and for this reason, the model is fit only to modulus data for systems containing chains of 16 monomers in length or longer, in which defects are likely to be spaced far apart from one another. The resulting fits are displayed in Figure 30, and suggest that the model is indeed capable of capturing the modulus behavior of the chains.

The analyses performed here show that axially loaded PPTA chains effectively bear the entire load in perfect crystals and therefore there the axial modulus is governed by the chain stiffness. The introduction of defects degrades the axial modulus and introduces a weak dependence on hydrogen bonds to the axial modulus as hydrogen bonds are involved in transferring load around chain ends. The model proposed by equation (6) describes this behavior adequately by considering chains as a series connection of perfect and defective (degraded by the presence of chainend defects) chains. The analysis also implies that the modulus does not depend on defect location as long as the defects are far enough apart as to be non-interacting, and this finding is consistent with the results in Figure 24 showing equal modulus for Variants I-III. In conjunction with the observation that crystallite stiffness gains are diminished after chains reach a length of about 64 monomers or longer, these results provide important insight into the relationship between chain length, defect concentration/distribution, and crystallite modulus. 


\section{Summary and conclusions}

This work provides an in-depth study of the mechanics of PPTA crystallites in aramid fibers via MD simulation. Chain-end defects, the most common and fundamental types of defect in aramid fiber microstructure, have been investigated to determine their influence on the behavior of the crystal structure near defect sites. The zone of influence of a defect is found to extend 8 monomers in both directions down the chain axis and one monomer ahead and behind the defect transversely. In constant strain-rate simulations of loading crystallites to failure, Type 2 bonds were observed to always fail first along the backbone of PPTA chains, identifying this bond as the weak link in the polymer chain, and both modulus and bond failure mechanisms were observed to be independent of strain-rate for the range of rates tested. The analysis of the influence of chain-end defects on the crystallite strength revealed: 1) that clustering of defects reduce crystallite strength and leads to an increased probability of failure via chain slippage and hydrogen bond rupture, and 2) randomly scattering chain-end locations throughout the crystallite minimizes the reduction in strength due to defects, provided the chain-ends do not interact with each other, and leads to crystallite failure dominated by primary bond rupture. An analysis of the influence of chain length and chain-end defect distribution on the crystallite modulus revealed that the modulus experiences dramatic improvement with increasing chain length for chains up to about 64 monomers in length, but this improvement plateaus significantly for chains longer than 64 monomers. Additionally, it was found that only the length of the chains (or equivalently, chain-end defect concentration) had an effect on the crystallite modulus; the modulus was practically independent of the distribution of the chain-end locations in the crystallite, as long as chain-ends were scattered sufficiently far from one another.

Collectively, these results offer a number of insights into how microstructural details of the crystallites translate to PPTA fiber properties. For example, the zone of influence studies help to explain how PPTA fibers retain such high strength and stiffness despite the presence of periodic defect planes spanning the length of the fiber. Essentially, the zone of influence of defects is so small that the crystallite strength is not significantly degraded despite the relatively narrow 20-40 nm (about 15-30 monomers) width of these defect cluster planes. The hydrogen bonds are 
strong enough to transfer load near a chain-end defect into adjacent chains, but also weak enough to rupture and accommodate crack tip blunting to mitigate chain rupture and crack propagation near defect sites. The simulation results also offer insights on how chain-end defect distribution affects fiber modulus and strength and how to improve on those properties, if desired: avoiding chain-end defect clusters will improve fiber strength, but has little influence on modulus. Modulus improvements should be sought through improving polymer chain alignment, since the simulation results here suggest that relative increase in modulus is minimal for chains exceeding 64 monomers in length, much shorter than the average chain length in typical manufactured aramid fibers. These findings also lay a foundation for establishing a multiscale constitutive model for a single PPTA fiber based on the mechanical properties of the underlying crystallites. In subsequent work, the strainrate sensitivity of the mechanical properties of PPTA crystallites will be analyzed in detail, providing further information for synthesizing such a constitutive model.

\section{Acknowledgments}

The authors with to thank Drs. Richard Gee and William Kuo at the Lawrence Livermore National Laboratory for many helpful conversations which helped to shape the direction of this research.

This work was performed under the auspices of the US Department of Energy by the Lawrence Livermore National Laboratory under contract DE-AC52-07NA27344.

\section{References}

1. Northolt, M. X-ray diffraction study of poly(p-phenylene terephthalamide) fibres. European Polymer Journal 10, 799-804 (1974).

2. Dobb, M., Johnson, D. \& Saville, B. Direct observation of structure in high-modulus aromatic fibers. Journal of Polymer Science: Polymer Symposia 58, 237-251 (1977).

3. Dobb, M., Johnson, D. \& Saville, B. Supramolecular structure of a high-modulus polyaromatic fiber (Kevlar 49). Journal of Polymer Science: Polymer Physics Edition 15, 2201-2211 (1977). 
4. Dobb, M., Johnson, D. \& Saville, B. Structural aspects of high modulus polyamide fibres. Philosophical Transactions of the Royal Society of London. Series A, Mathemetical and Physical Sciences 294, 483-485 (2013).

5. Panar, M., Avakian, P., Blume, R., Gardner, K., Gierke, T. \& Yang, H. Morphology of Poly (p-Phenylene Terephthalamide) Fibers. Journal of Polymer Science: Polymer Physics Edition 21, 1955-1969 (1983).

6. Morgan, R., Pruneda, C. \& Steele, W. The Relationship between the Physical Structure and the Microscopic Deformation and Failiure Processes of Poly(pPhenylene Terephthalamide) Fibers. Journal of Polymer Science: Polymer Physics Edition 21, 1757-1783 (1983).

7. Li, L., Allard, L. \& Bigelow, W. On the morphology of aromatic polyamide fibers (Kevlar, Kevlar-49, and PRD-49). Journal of Macromolecular Science, Part B 22, 269-290 (1983).

8. Northolt, M. \& van Aartsen, J. Chain orientation distribution and elastic properties of poly (p-phenylene terephthalamide), a "rigid rod" polymer. Journal of Polymer Science: Polymer Symposium 58, 283-296 (1977).

9. Northolt, M. Tensile deformation of poly(p-phenylene terephthalamide) fibres, an experimental and theoretical analysis. Polymer 21, 1199-1204 (1980).

10. Northolt, M. \& van der Hout, R. Elastic extension of an oriented crystalline fibre. Polymer 26, 310-316 (1985).

11. Rao, Y., Waddon, A. \& Farris, R. Structure-property relation in poly (p-phenylene terephthalamide) (PPTA) fibers. Polymer 42, 5937-5946 (2001).

12. Depner, M. \& Schfirmann, B. Computer simulation of aromatic polyesters including molecular dynamics. Polymer 33, 398-404 (1990).

13. Tashiro, K., Kobayashi, M. \& Tadokoro, H. Elastic Moduli and Molecular Structures of Several Crystalline Polymers, Including Aromatic Polyamides. Macromolecules 10, 413-420 (1977).

14. Lacks, D. \& Rutledge, G. Thermal Expansion and Temperature Dependence of Elastic Moduli of Aromatic Polyamides. Macromolecules 27, 7197-7204 (1994).

15. Tadokoro, H. Structure and properties of crystalline polymers. Polymer 25, 147-164 (1984). 
16. Lacks, D. Molecular simulation of compressive failure in poly(p-phenylene teraphthalamide) crystals. Journal of Materials Science 31, 5885-5889 (1996).

17. Grujicic, M., Bell, W., Glomski, P., Pandurangan, B., Yen, C.-F. \& Cheeseman, B. Filament-level modeling of aramid-based high-performance structural materials. Journal of Materials Engineering and Performance 20, 1401-1413 (2011).

18. Grujicic, M., Glomski, P., Pandurangan, B., Bell, W., Yen, C.-F. \& Cheeseman, B. Multi-length scale computational derivation of Kevlar ${ }^{\circledR}$ yarn-level material model. Journal of Materials Science 46, 4787-4802 (2011).

19. Grujicic, M., Pandurangan, B., Snipes, J., Yen, C.-F. \& Cheeseman, B. Multi-Length Scale-Enriched Continuum-Level Material Model for Kevlar®-Fiber-Reinforced Polymer-Matrix Composites. Journal of Materials Engineering and Performance 22, 681-695 (2013).

20. Grujicic, M., Yavari, R., Ramaswami, S., Snipes, J., Yen, C.-F. \& Cheeseman, B. Molecular-Level Study of the Effect of Prior Axial Compression/Torsion on the AxialTensile Strength of PPTA Fibers. Journal of Materials Engineering and Performance 22, 3269-3287 (2013).

21. Grujicic, M., Ramaswami, S., Snipes, J., Yavari, R., Yen, C.-F. \& Cheeseman, B. Formation and Propagation, of Single p-Phenylene Terephthalamide (PPTA) Fibers. Advances in Materials Science and Engineering 2013, 1-15 (2013).

22. Grujicic, M., Yavari, R., Snipes, J., Ramaswami, S., Yen, C.-F. \& Cheeseman, B. The effect of plain-weaving on the mechanical properties of warp and weft p-phenylene terephthalamide (PPTA) fibers/yarns. Journal of Materials Science 49, 8272-8293 (2014).

23. van Duin, A., Dasgupta, S., Lorant, F. \& Goddard III, W. ReaxFF: A Reactive Force Field for Hydrocarbons. The Journal of Physical Chemistry A 105, 9396-9409 (2001).

24. van Duin, A., Strachan, A., Stewman, S., Zhang, Q., Xu, X. \& Goddard III, W. ReaxFF SiO Reactive Force Field for Silicon and Silicon Oxide Systems. Journal of Physical Chemistry A 107, 3803-3811 (2003).

25. Shin, Y., Kwak, H., Zou, C., Vasenkov, A. \& van Duin, A. Development and Validation of a ReaxFF Reactive Force Field for Fe/Al/Ni Alloys: Molecular Dynamics Study of Elastic Constants, Diffusion, and Segregation. The Journal of Physical Chemistry A 116, 12163-12174 (2012). 
26. Nielson, K., van Duin, A., Oxgaard, J., Deng, W.-Q. \& Goddard III, W. Development of the ReaxFF reactive force field for describing transition metal catalyzed reactions, with application to the initial stages of the catalytic formation of carbon nanotubes. Journal of Physical Chemistry A 109, 493-499 (2005).

27. Liu, L., Liu, Y., Zybin, S., Sun, H. \& Goddard III, W. ReaxFF-lg: Correction of the ReaxFF reactive force field for London dispersion, with applications to the equations of state for energetic materials. The Journal of Physical Chemistry A 115, 1101611022 (2011).

28. Jensen, B., Wise, K. \& Odegard, G. The effect of time step, thermostat, and strain rate on ReaxFF simulations of mechanical failure in diamond, graphene, and carbon nanotube. Journal of Computational Chemistry 36, 1587-1596 (2015).

29. Sen, D., Novoselov, K., Reis, P. \& Buehler, M. Tearing Graphene Sheets From Adhesive Substrates Produces Tapered Nanoribbons. Small 6, 1108-1116 (2010).

30. Kim, K., Artyukhov, V., Regan, W., Liu, Y., Crommie, M., Yakobson, B. \& Zettl, A. Ripping graphene: Preferred directions. Nano Letters 12, 293-297 (2012).

31. Cranford, S. \& Buehler, M. Mechanical properties of graphyne. Carbon 49, 4111-4121 (2011).

32. Cranford, S., Brommer, D. \& Buehler, M. Extended graphynes: simple scaling laws for stiffness, strength and fracture. Nanoscale 4, 7797-7809 (2012).

33. Buehler, M., van Duin, A. \& Goddard III, W. Multiparadigm Modeling of Dynamical Crack Propagation in Silicon Using a Reactive Force Field. Physical Review Letters 96, 095505 (2006).

34. Yilmaz, D. Modeling failure mechanisms of poly ( $\mathrm{p}$-phenylene terephthalamide) fiber using reactive potentials. Computational Materials Science 109, 183-193 (2015).

35. Strachan, A., van Duin, A., Chakraborty, D., Dasgupta, S. \& Goddard III, W. Shock waves in high-energy materials: the initial chemical events in nitramine RDX. Physical Review Letters 91, 098301 (2003).

36. Sun, H., Mumby, S., Maple, J. \& Hagler, A. An ab Initio CFF93 All-Atom Force Field for Polycarbonates. Journal of the American Chemical Society 116, 2978-2987 (1994). 
37. Rutledge, G. \& Suter, U. Detailed Atomistic Simulation of Oriented Pseudocrystalline Polymers and Application to a Stiff-Chain Aramid. Macromolecules 24, 1921-1933 (1991).

38. Rutledge, G. \& Suter, U. Calculation of mechanical properties of poly(p-phenylene terephthalamide) by atomistic modelling. Polymer 32, 2179-2189 (1991).

39. Sun, H. COMPASS: An ab Initio Force-Field Optimized for Condensed-Phase Applications - Overview with Details on Alkane and Benzene Compounds. Journal of Physical Chemistry B 102, 7338-7364 (1998).

40. Plimpton, S. Fast Parallel Algorithms for Short-Range Molecular Dynamics. Journal of Computational Physics 117, 1-19 (1995).

41. Accelrys Software Inc. Materials Studio San Diego, 2011.

42. Nosé, S. A unified formulation of the constant temperature molecular dynamics methods. The Journal of Chemical Physics 81, 511-519 (1984).

43. Hoover, W. Canonical dynamics: Equilibrium phase-space distributions. Physical Review A 31, 1695-1697 (1985).

44. Martyna, G. J., Tobias, D. J. \& Klein, M. L. Constant pressure molecular dynamics algorithms. The Journal of Chemical Physics 101, 4177-4189 (1994).

45. Hossain, D., Tschopp, M., Ward, D., Bouvard, J., Wang, P. \& Horstemeyer, M. Molecular dynamics simulations of deformation mechanisms of amorphous polyethylene. Polymer 51, 6071-6083 (2010).

46. Rappé, A. \& Goddard III, W. Charge Equilibration for Molecular Dynamics Simulations. The Journal of Physical Chemistry 95, 3358-3363 (1991).

47. Nakano, A. Parallel multilevel preconditioned conjugate-gradient approach to variable-charge molecular dynamics. Computer Physics Communications 104, 5969 (1997).

48. Aktulga, H., Pandit, S., van Duin, A. \& Grama, A. Reactive Molecular Dynamics: Numerical Methods and Algorithmic Techniques. SIAM Journal on Scientific Computing 34, C1-C23 (2012).

49. Hockney, R. \& Eastwood, J. Computer Simulation Using Particles (Taylor \& Francis Group, New York, NY, 1988). 
50. Tsai, D. H. The virial theorem and stress calculation in molecular dynamics. The Journal of Chemical Physics 70, 1375 (1979).

51. Yang, X. \& Hsu, S. Application of Molecular Simulation Technique To Calculate Structure and Define Deformation Mechanisms of High-Performance Polymers. Macromolecules 24, 6680-6685 (1991).

52. Gaymans, R., Tijssen, J., Harkema, S. \& Bantjes, A. Elastic modulus in the crystalline region of poly (p-phenylene terephthalamide). Polymer 17, 517-518 (1976).

53. Barton, R. Paracrystallinity-modulus relationships in Kevlar aramid fibers. Journal of Macromolecular Science, Part B B24, 119-130 (1985).

54. Young, R., Lu, D., Day, R., Knoff, W. \& Davis, H. Relationship between structure and mechanical properties for aramid fibres. Journal of Materials Science 27, 5431-5440 (1992).

55. Ii, T., Tashiro, K., Kobayashi, M. \& Tadokoro, H. X-ray study of lattice tensile properties of fully extended aromatic polyamide fibers over a wide temperature range. Macromolecules 20, 347-351 (1987).

56. Buckley, C. The Ultimate Strength and Stiffness of Polymers. Annu. Rev. Mater. Sci. 25, 295-323 (1995).

57. Low, I.-M. \& Mai, Y.-W. in Handbook of Ceramics and Composites (ed Cheremisinoff, N.) 105-160 (Marcel Dekker, Inc., New York, NY, 1992).

58. Chenoweth, K., van Duin, A. \& Goddard III, W. ReaxFF reactive force field for molecular dynamics simulations of hydrocarbon oxidation. The Journal of Physical Chemistry A 112, 1040-1053 (2008).

59. Luzar, A. \& Chandler, D. Structure and hydrogen bond dynamics of water-dimethyl sulfoxide mixtures by computer simulations. The Journal of Chemical Physics 98, 8160-8173 (1993).

60. Chandra, A. Dynamical Behavior of Anion-Water and Water-Water Hydrogen Bonds in Aqueous Electrolyte Solutions: A Molecular Dynamics Study. Journal of Physical Chemistry B 107, 3899-3906 (2003).

61. Ahmed, D., Hongpeng, Z., Haijuan, K., Jing, L., Yu, M. \& Muhuo, Y. Microstructural Developments of Poly (p-phenylene terephthalamide) Fibers During Heat Treatment Process: A Review. Materials Researh 17, 1180-1200 (2014). 
62. O'Connor, T. C. \& Robbins, M. O. Chain Ends and the Ultimate Strength of Polyethylene Fibers. ACS Macro Letters 5, 263-267 (2016). 


\section{Appendix}

\section{A Derivation of elastic moduli for crystallite with non-interacting defects}

Consider a crystallite consisting of chains of length $L_{c}$, with chain-end defects randomly distributed throughout the crystallite. It is assumed that the defect concentration is sparse enough that the zones of influence of the individual defect sites (as discussed in Section 4) do not overlap with one another. The collective effect of defects in the crystallite renders individual chains dividable into two distinct regions: one which is entirely unaffected by the defects in the system due to being sufficiently far away from any chain-end defects sites, and another which experiences a combination of stressed and relaxed regions (not necessarily adjacent to one another). The total length of the uninfluenced part of the chain is denoted $L_{1}$, and the total length of the influenced part of the chain is denoted $L_{2}$, with $L_{c}=L_{1}+L_{2}$. Each part of the chain has an associated spring stiffness, such that $k_{1}$ represents the nominal stiffness of a chain uninfluenced by defects, and $k_{2}$ represents the degraded stiffness of the chain which is influenced by defects. The effective stiffness $k_{c}$ of this series of two chains is given by

$$
k_{c}=\frac{1}{\frac{1}{k_{1}}+\frac{1}{k_{2}}}=\frac{k_{1} k_{2}}{k_{1}+k_{2}} .
$$

Assuming each chain covers the same cross-sectional area, an effective Young's modulus can be computed as

$$
\frac{E}{L_{c}}=\frac{\frac{E_{1} E_{2}}{L_{1} L_{2}}}{\frac{E_{1}}{L_{1}}+\frac{E_{2}}{L_{2}}} .
$$

The ratio of chain stiffness to the stiffness in a perfect crystal can then be expressed as

$$
\frac{E}{E_{1}}=\frac{L_{c} E_{2}}{L_{2} E_{1}+L_{1} E_{2}} .
$$

Defining chain length fractions $f_{i}=\frac{L_{i}}{L_{c}}, i=1,2$, equation (A.3) can be rewritten as

$$
\frac{E}{E_{1}}=\frac{1}{f_{1}+f_{2} \frac{E_{1}}{E_{2}}} .
$$


Since chain length fractions sum to 1 and $f_{2}=\frac{L_{2}}{L_{c}}$, equation (A.4) can be rewritten once more as

$$
\frac{E}{E_{1}}=\frac{1}{1+\frac{1}{L_{c}}\left[L_{2}\left(\frac{E_{1}}{E_{2}}-1\right)\right]} \text {. }
$$

In equation (A.5), $E_{1}$ (modulus of a perfect crystallite), $E_{2}$ (modulus of the region weakened by non-interacting defects in a crystallite), and $L_{2}$ (length of chain influenced by non-interacting defects in a crystallite) are physical properties of the crystallite and PPTA chains, and therefore can be treated as constants. As a result, the ratio of overall crystal modulus to perfect crystal modulus can be expressed more concisely as

$$
\frac{E}{E_{1}}=\frac{1}{1+\frac{\phi}{L_{c}}},
$$

where $\phi=\left(\frac{E_{1}}{E_{2}}-1\right) L_{2}$ and can be treated as a material constant. 

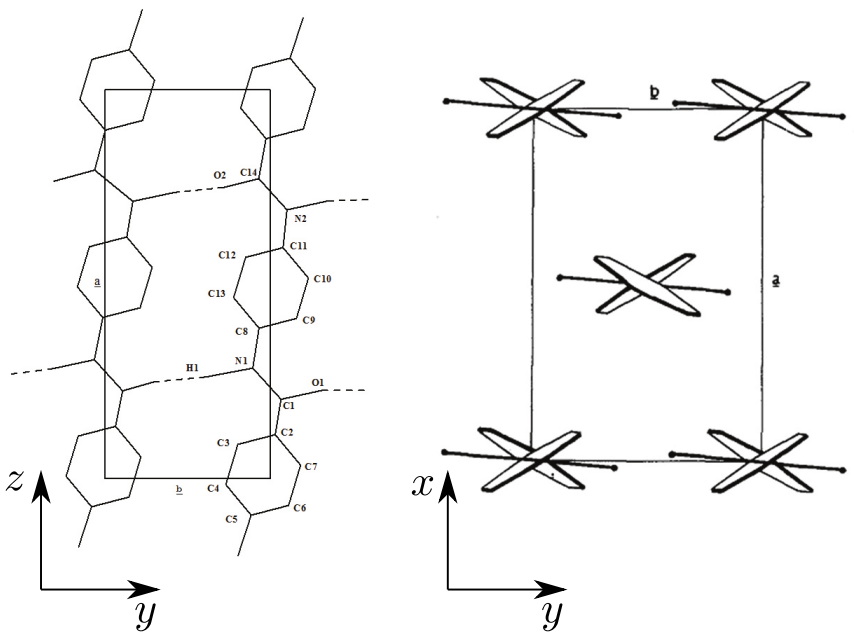

Figure 1: Crystalline unit cell for PPTA [1]. The $x, y$, and $z$ Cartesian axes correspond to the $a, b$, and $c$ crystalline unit cell dimensions, respectively. The $z$ axis corresponds to the fiber axis. 


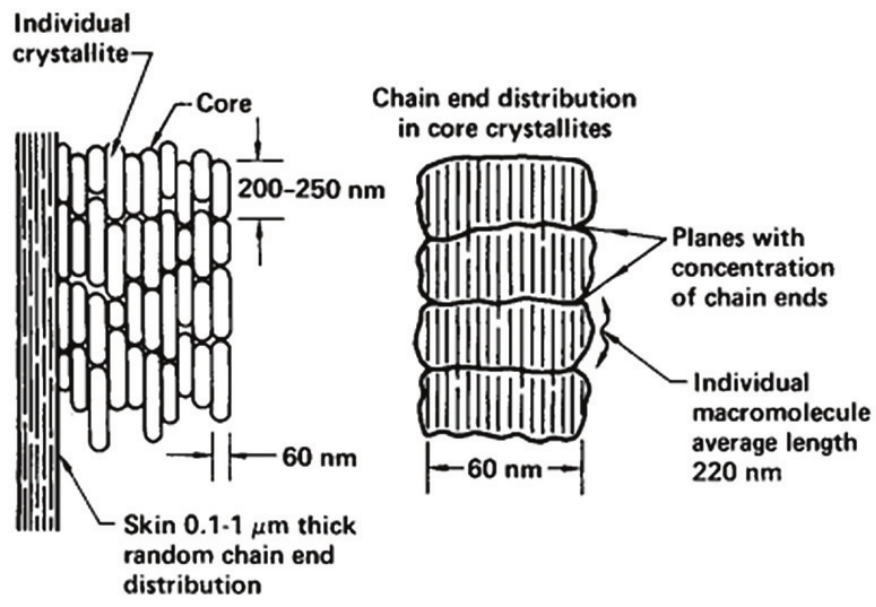

Figure 2: Aramid fiber crystallite morphology model proposed by Morgan et al. [6]. 


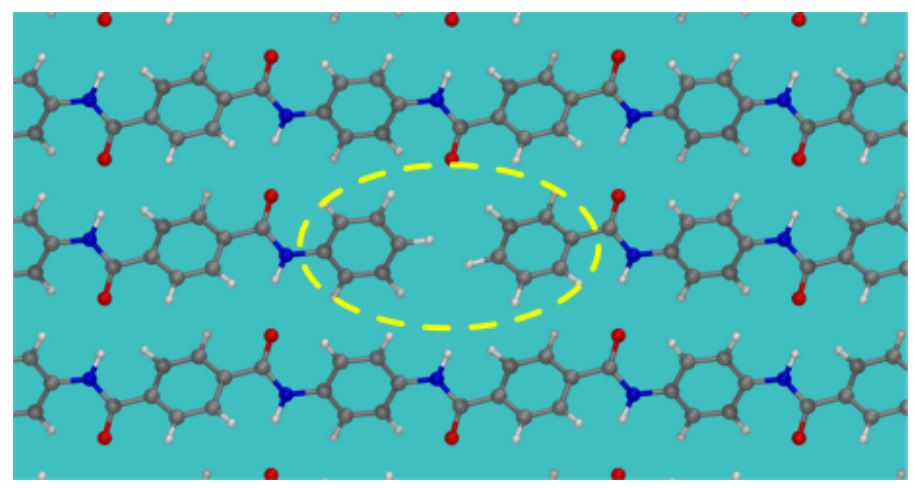

Figure 3: A chain-end defect is introduced by removing the bonds linking two aromatic rings, and capping the rings with hydrogen atoms. C, H, O and $\mathrm{N}$ atoms are colored gray, white, red, and blue, respectively. 


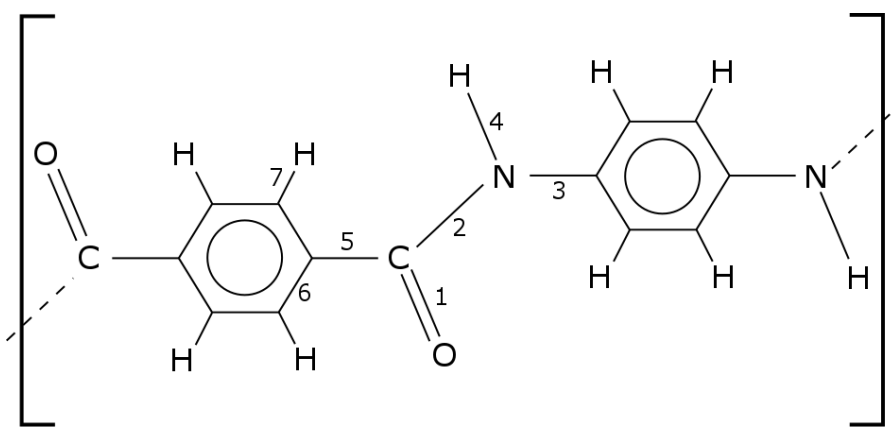

Figure 4: Diagram of a single PPTA monomer. Dotted-line bonds indicate periodic repetition of the monomer in a long chain. 


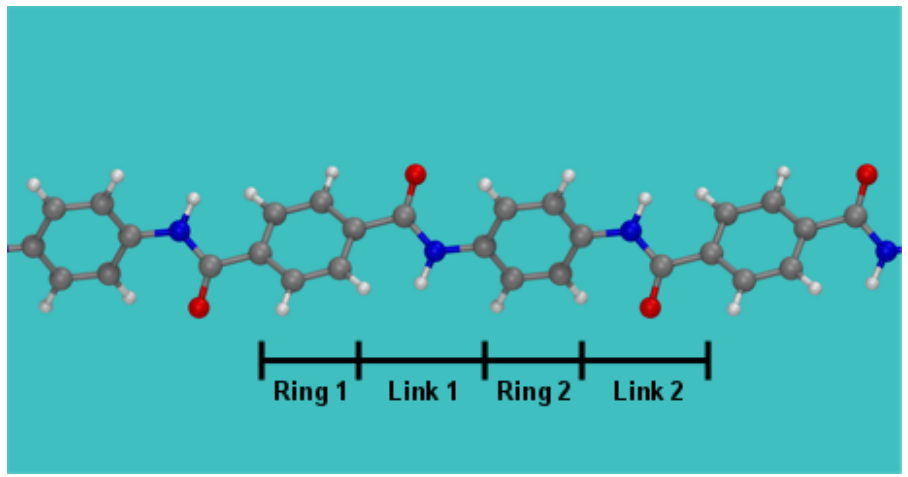

Figure 5: Breakdown of a monomer into four major components, two aromatic rings and two links. Each link consists of three covalent bonds (labeled 2, 3, and 5 in Figure 4). 


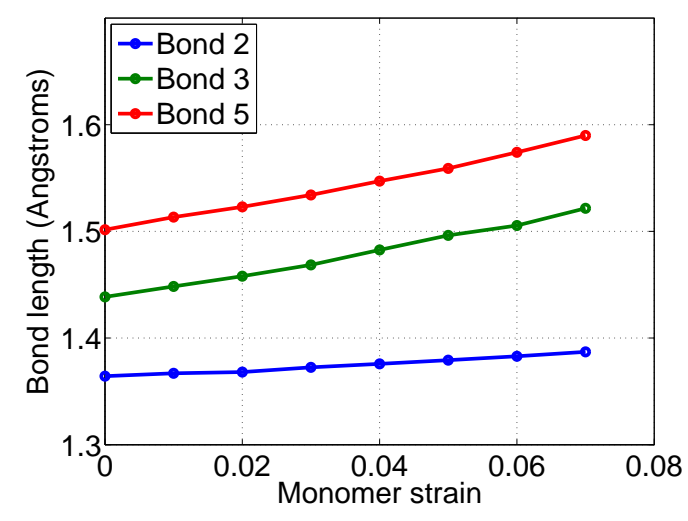

(a) PCFF

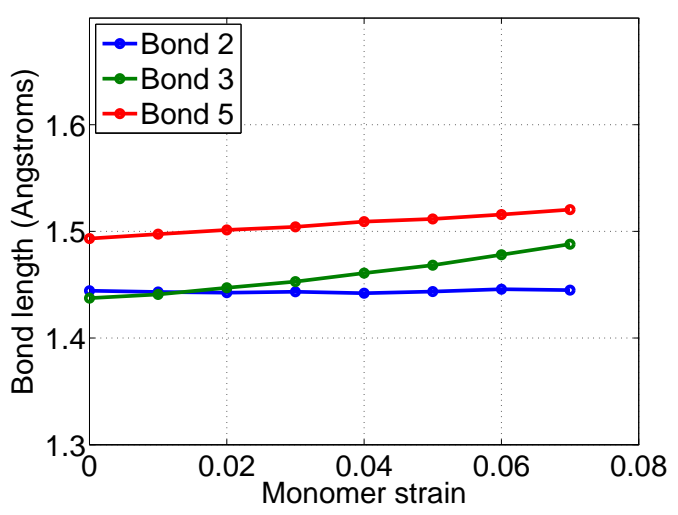

(b) REAXFF

Figure 6: Bond length versus monomer strain. 


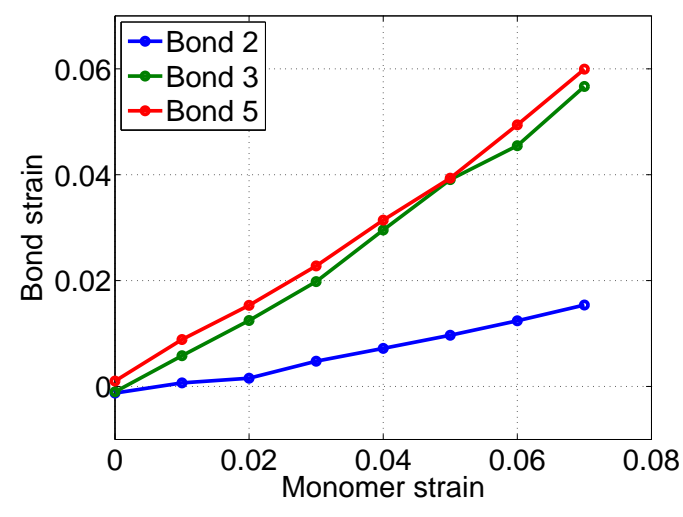

(a) $\mathrm{PCFF}$

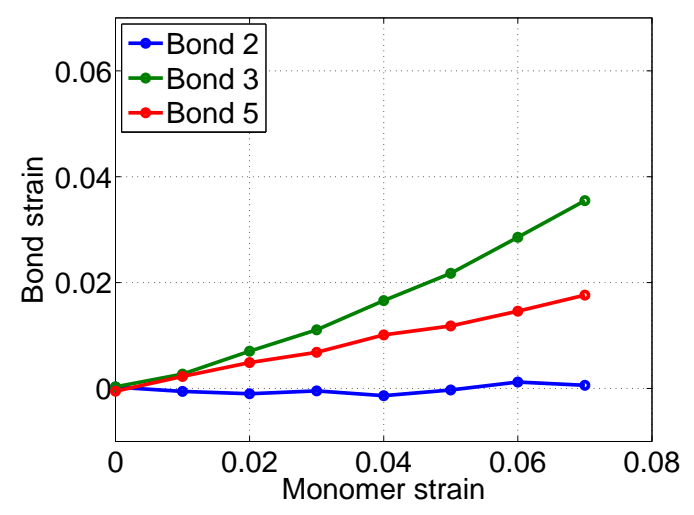

(b) REAXFF

Figure 7: Bond strain versus monomer strain. 


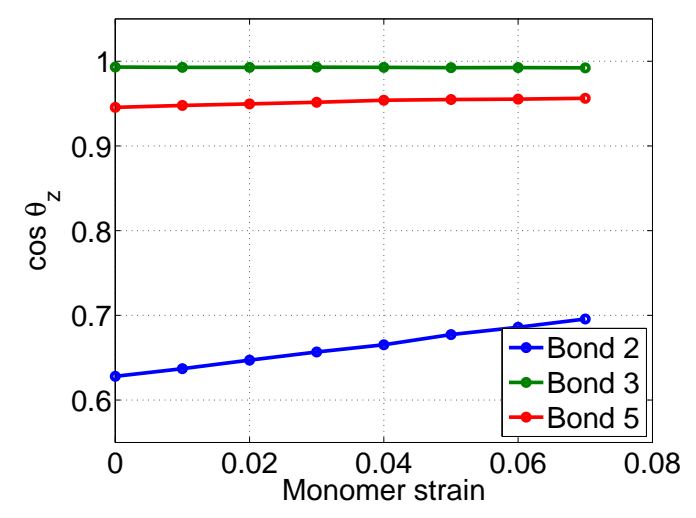

(a) $\mathrm{PCFF}$

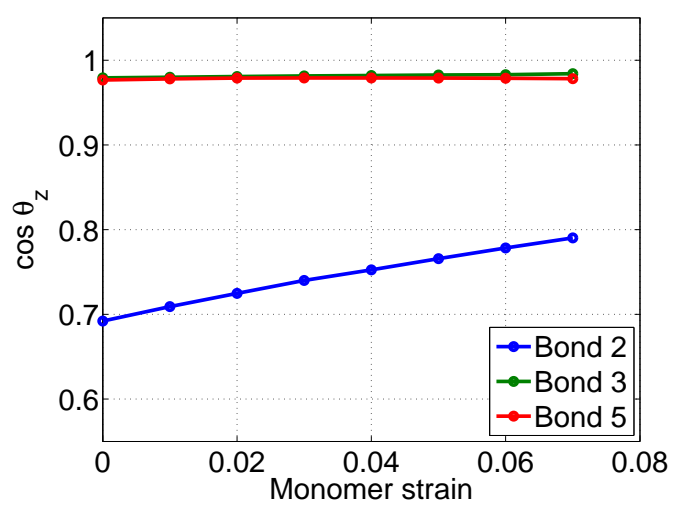

(b) REAXFF

Figure 8: Bond orientation versus monomer strain. 


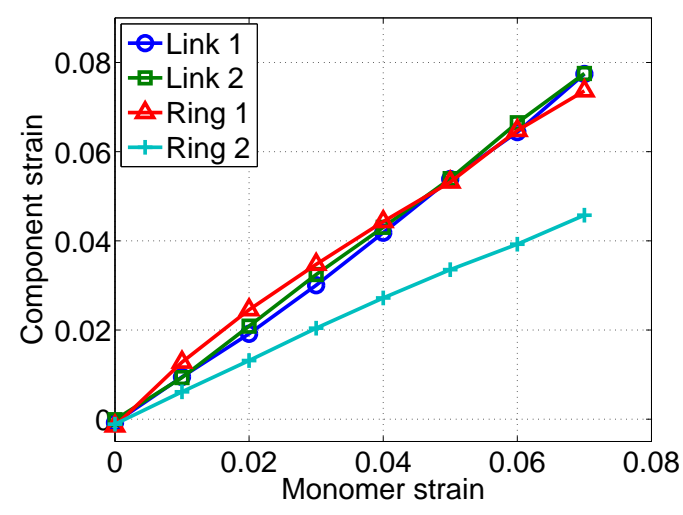

(a) PCFF

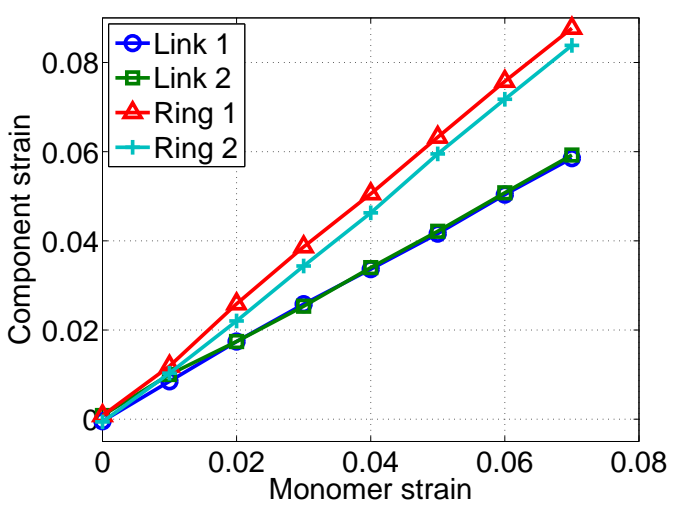

(b) REAXFF

Figure 9: Strain in the two links and two rings comprising a single monomer, versus monomer strain. Component labels follow those in Figure 5. 

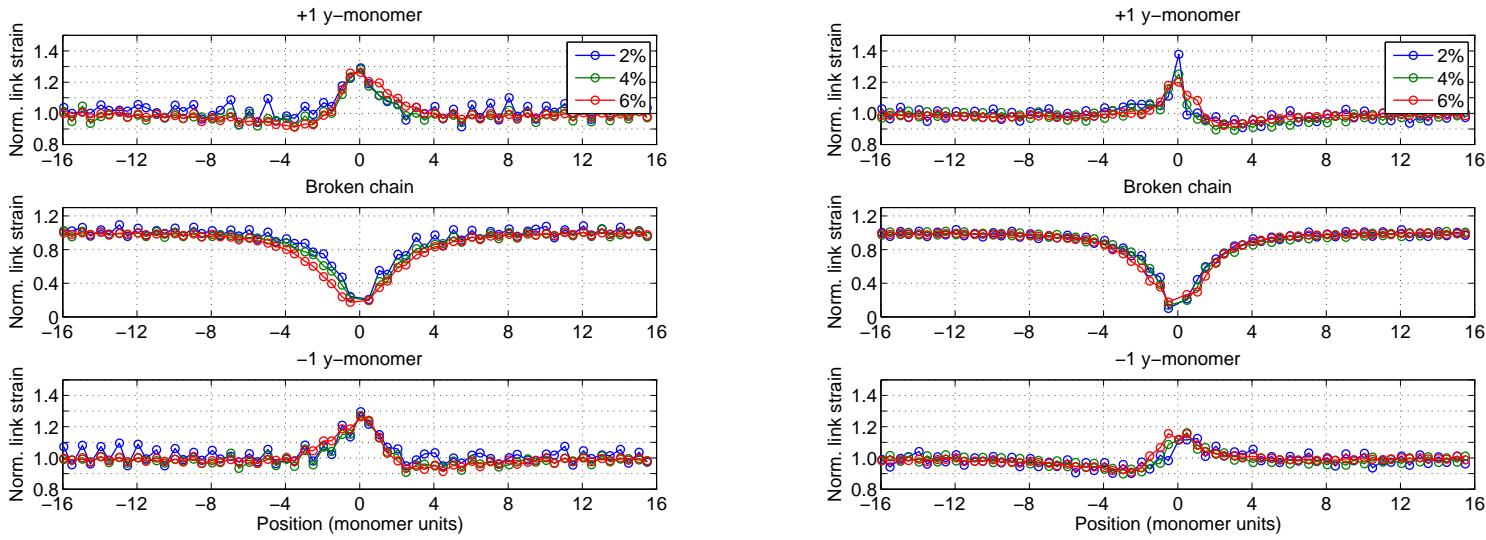

(a) PCFF

(b) ReaxFF

Figure 10: Normalized link strain (relative to nominal levels in a perfect crystal) in PPTA chains for a system containing a single chain-end defect at monomer position 0 . 

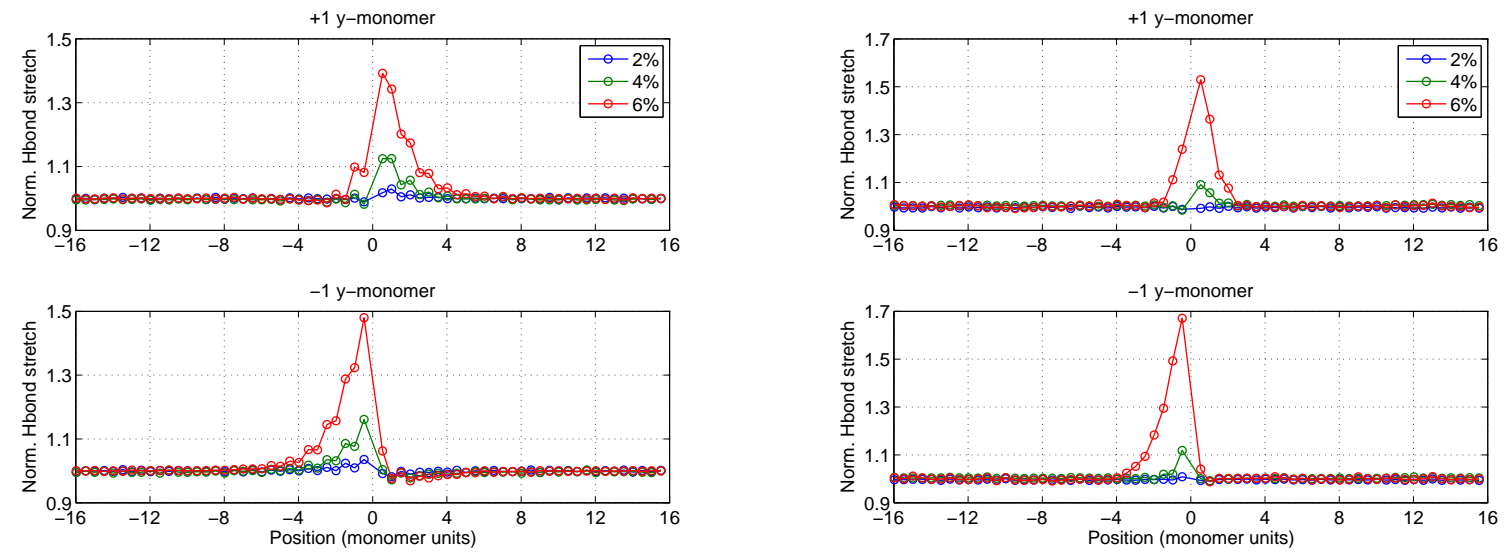

(a) $\mathrm{PCFF}$

(b) REAxFF

Figure 11: Normalized hydrogen bond stretch (relative to nominal levels in a perfect crystal) in PPTA chains for a system containing a single chain-end defect located at monomer position 0 . 


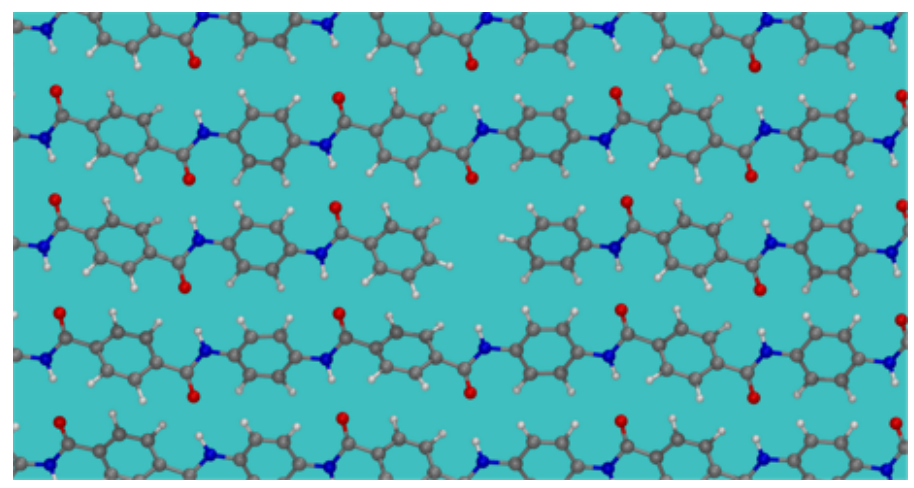

(a) $2 \%$ crystal strain

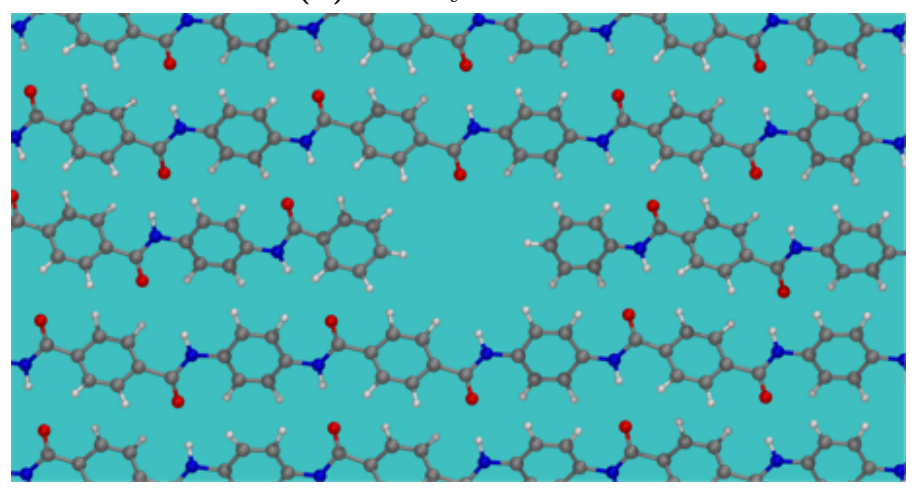

(b) $6 \%$ crystal strain

Figure 12: Visualization of the molecular deformation near the site of the chain-end defect, as predicted by PCFF. Note the increased stretch in hydrogen bonds near the defect, which occurs due to relaxation (shortening) of the broken chain relative to the adjacent chains. 

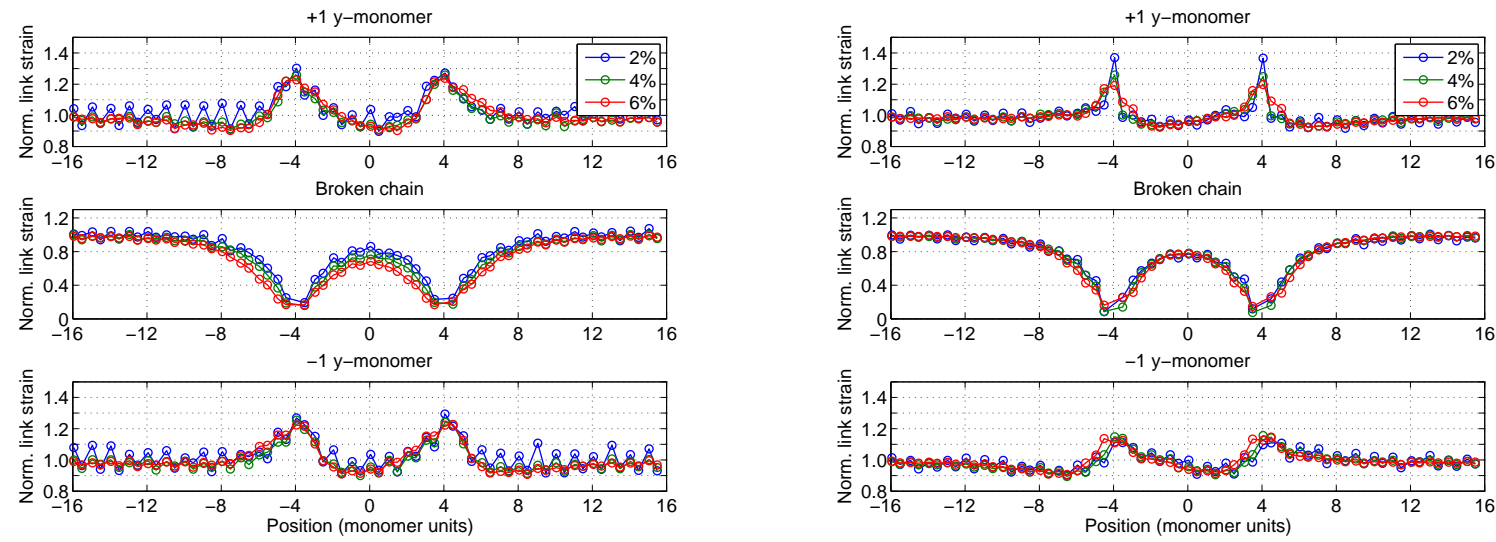

(a) PCFF

(b) REAxFF

Figure 13: Normalized link strain (relative to nominal levels in a perfect crystal) in PPTA chains for a system with two chain-end defects at monomer positions +4 and -4 . 


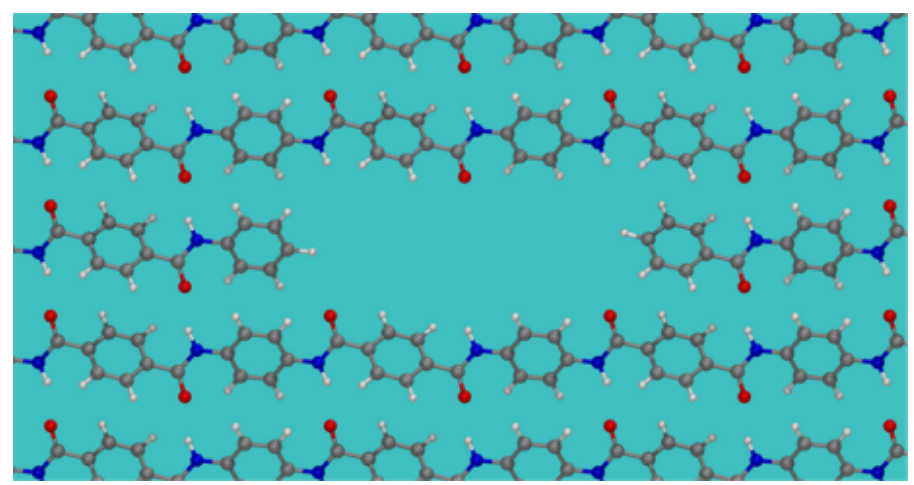

Figure 14: Visualization of a single molecule with three consecutive chain-end defects. 

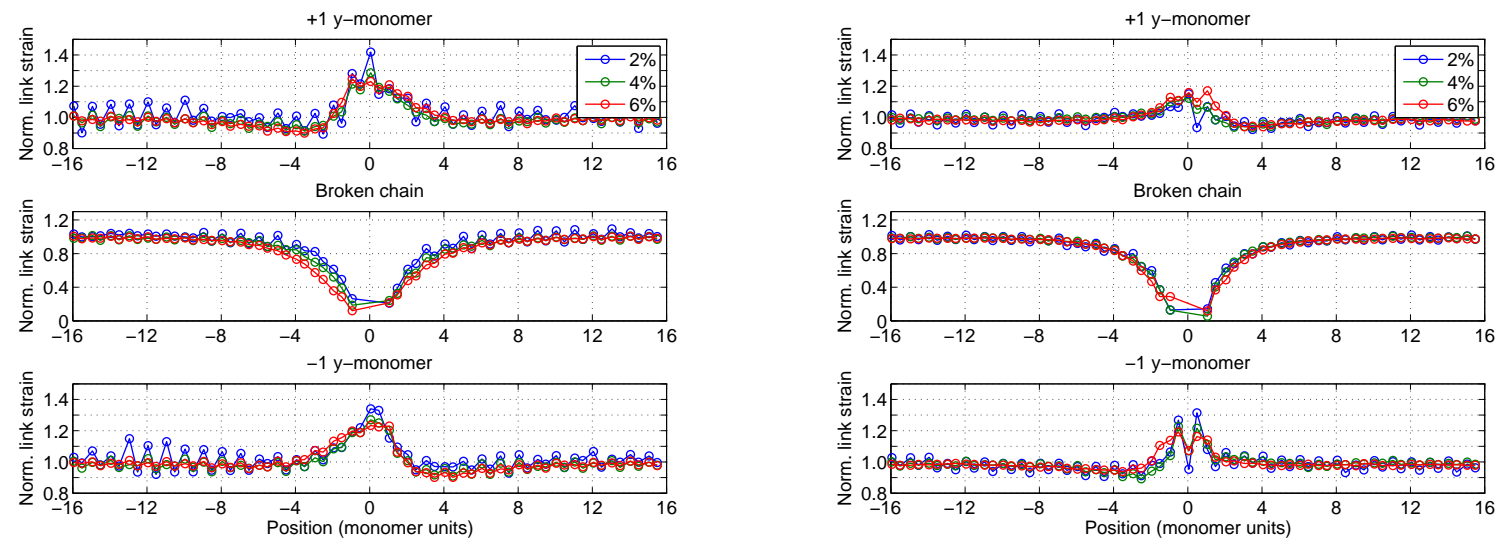

(a) $\mathrm{PCFF}$

(b) ReaxFF

Figure 15: Normalized link strain (relative to nominal levels in a perfect crystal) in PPTA chains for a system with three consecutive missing links in a single molecule. 


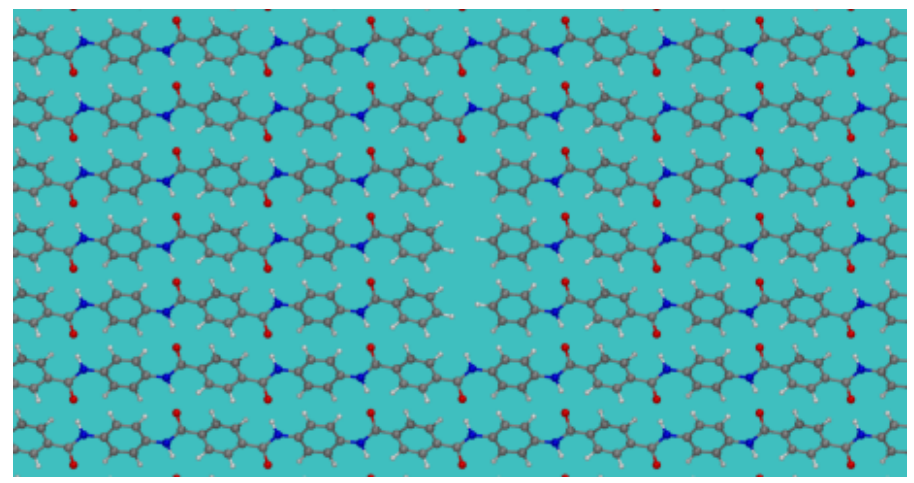

(a) $0 \%$ crystallite strain

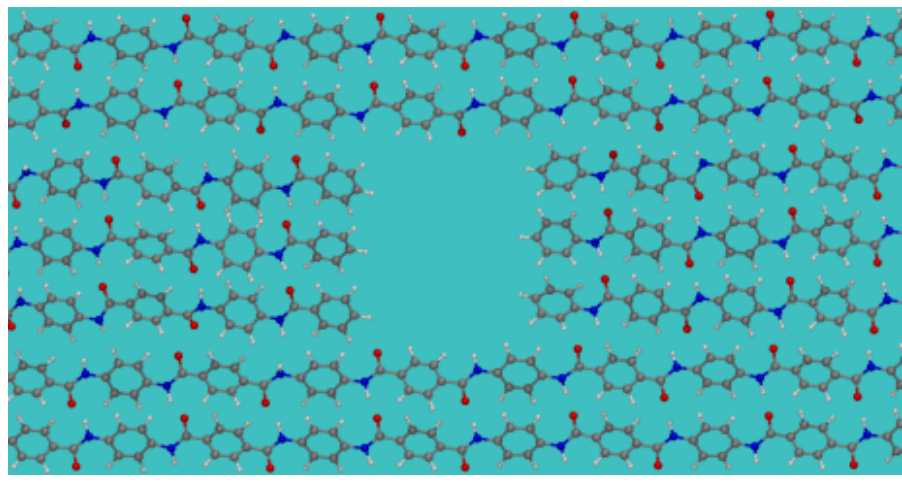

(b) $6 \%$ crystallite strain

Figure 16: Visualization of a crack-like defect resulting from three adjacent molecules with defects at the same axial position. Figure (a) shows the crack at equilibrium $(0 \%$ strain), and Figure (b) highlights the hydrogen bond failure that occurs for chains near the crack tip when the crystal is stretched to $6 \%$ strain, as predicted by REAxFF 

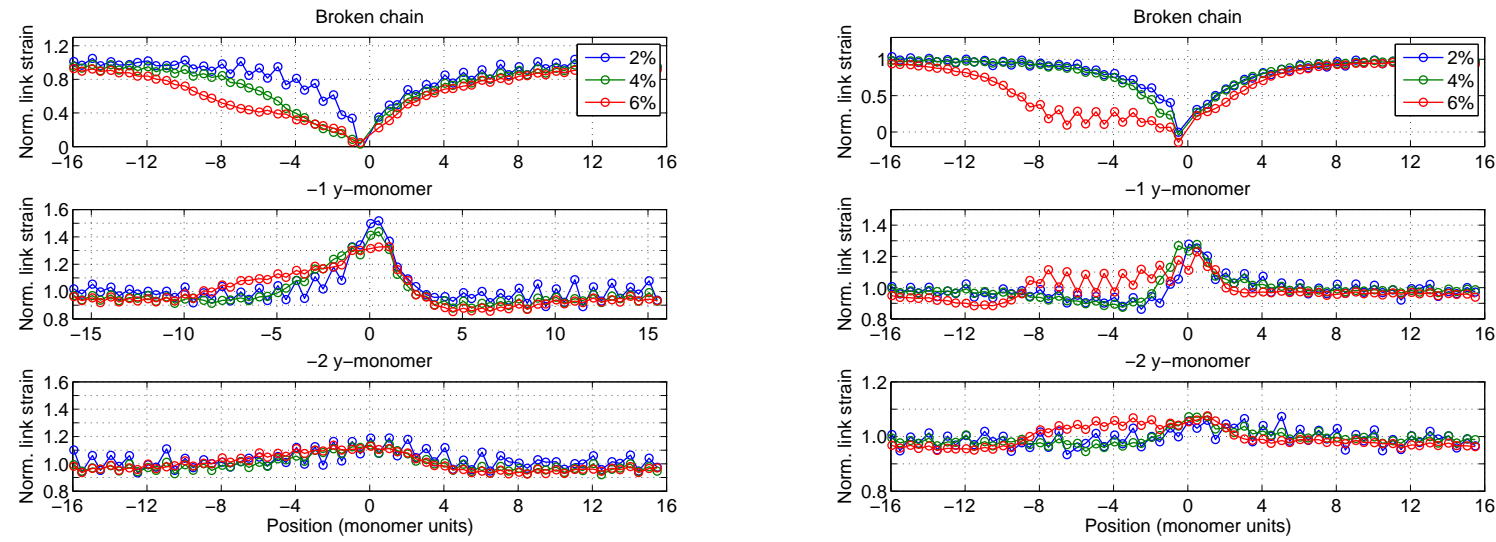

(a) PCFF

(b) REAXFF

Figure 17: Normalized link strain (relative to nominal levels in a perfect crystal) in PPTA chains for a system with a small crack, as illustrated in Figure 16a. 


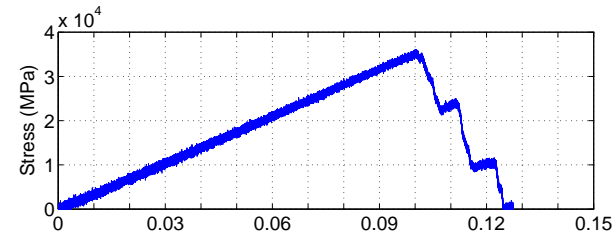

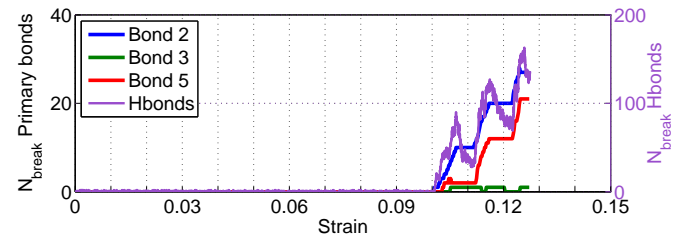

(a)

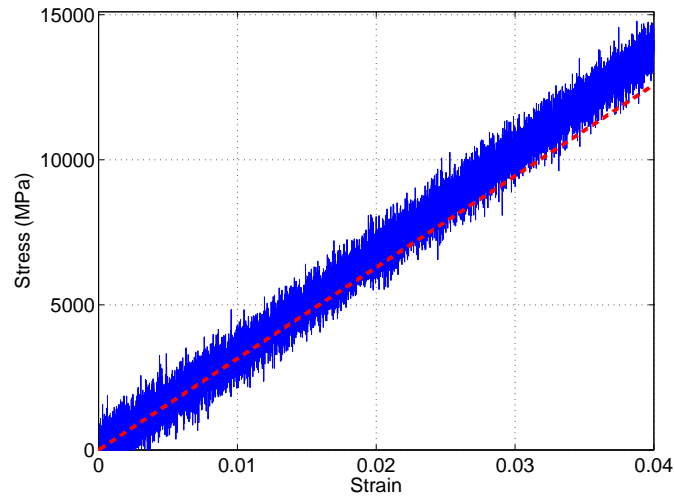

(b)

Figure 18: (a) Stress-strain curve and bond break history for the $4 \times 4 \times 8$ unit cell system loaded at $\dot{\epsilon}_{z z}=1.0 \times 10^{9} \mathrm{~s}^{-1}$; (b) Detailed view of low-strain regime, highlighting the increase in modulus relative to the initial (red dashed line) modulus. 


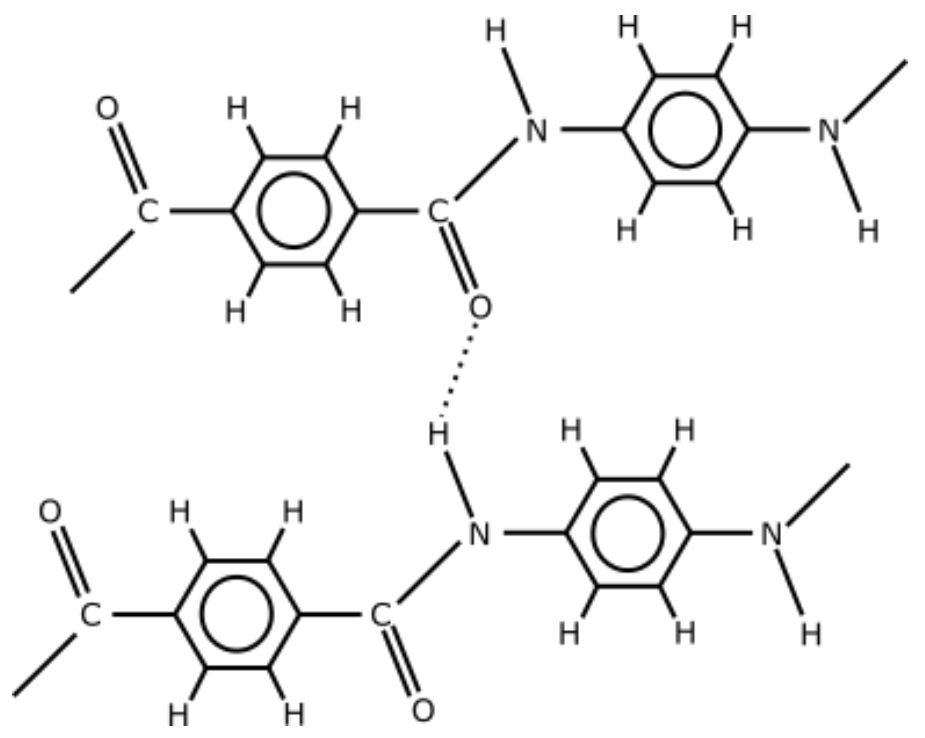

Figure 19: Diagram of a hydrogen bond between adjacent PPTA chains. The hydrogen bond length $d_{h b}$ is defined as the distance $\mathrm{O}-\mathrm{N}$ while the hydrogen bond angle $\theta_{h b}$ equals $\angle \mathrm{O}-\mathrm{H}-\mathrm{N}$. 

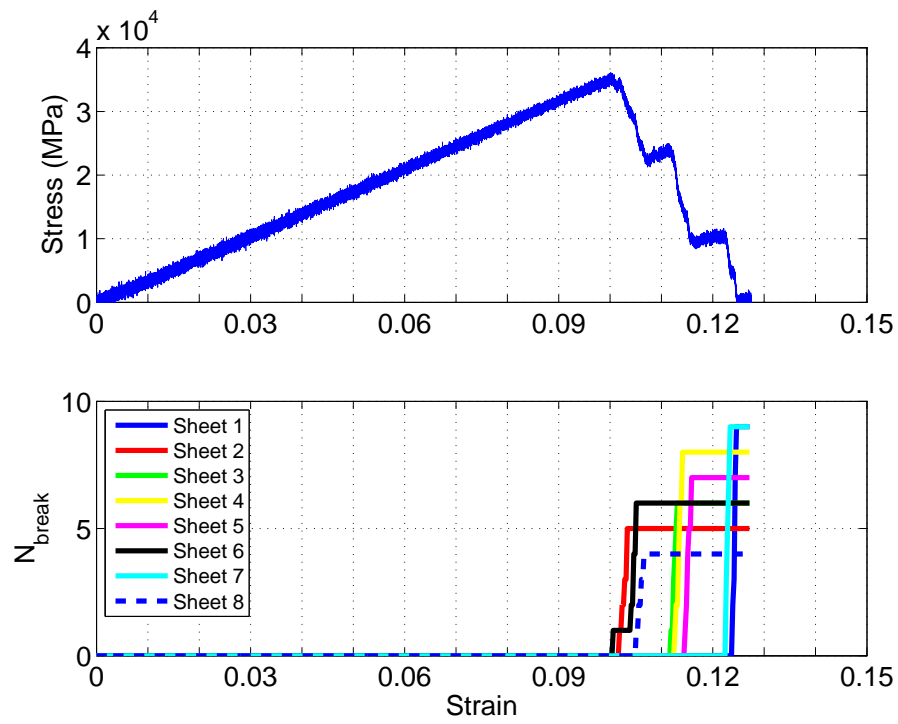

Figure 20: The stress-strain and bond break history plot from Figure 18 for the $4 \times 4 \times 8$ unit cell system is reproduced, but bond breaks are organized by hydrogen-bonded sheet. 


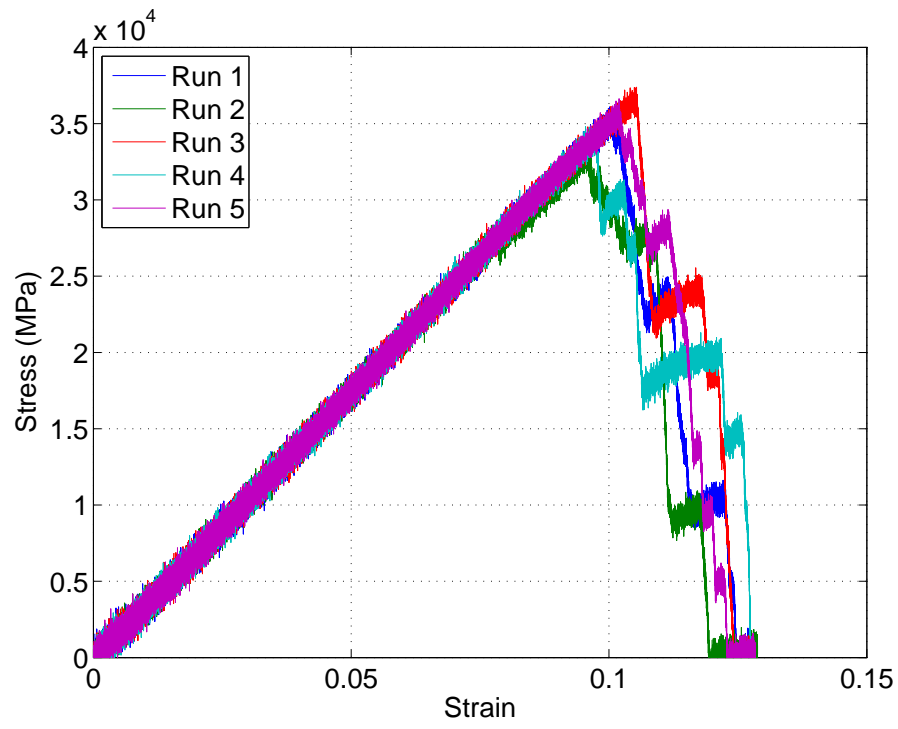

Figure 21: Simulation at strain rate $\dot{\epsilon}_{z z}=1.0 \times 10^{9} \mathrm{~s}^{-1}$ for five different equilibrated starting points of the $4 \times 4 \times 8$ unit cell system. The difference in failure strain between runs highlights the stochastic nature of the failure response. 

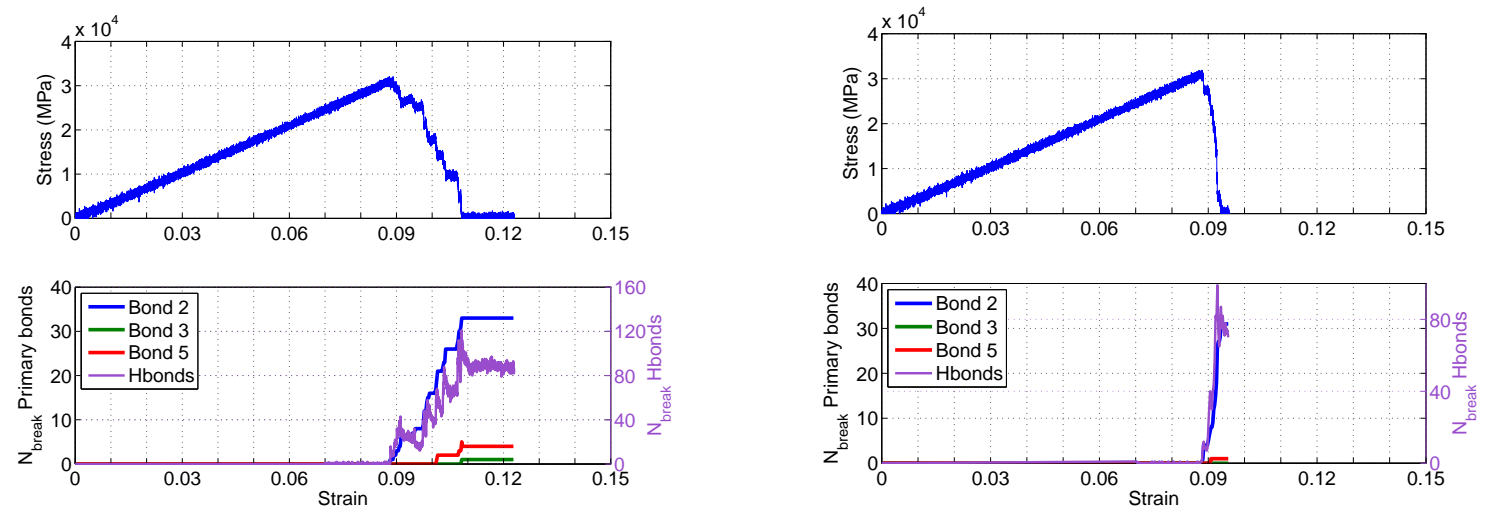

(a) $\dot{\epsilon}_{z z}=2.0 \times 10^{8} \mathrm{~s}^{-1}$

(b) $\dot{\epsilon}_{z z}=4.0 \times 10^{7} \mathrm{~s}^{-1}$

Figure 22: Stress-strain response and bond break history for two different strain rates of the $4 \times 4 \times 8$ unit cell system. 


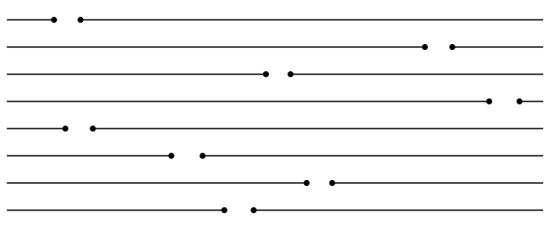

(a) Variant I: chain-ends distributed randomly.

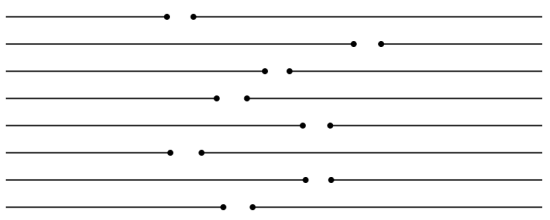

(b) Variant II: chain-ends lie within \pm 10 monomers of the center plane.

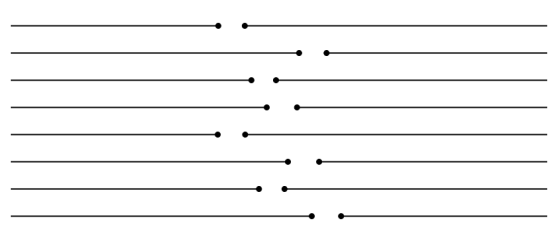

(c) Variant III: chain-ends lie within \pm 4 monomers of the center plane.

Figure 23: Diagram of chain-end locations for Variants I, II, and III (not to scale). Each diagram shows a representative single sheet of 8 chains and each simulation cell consists of $8 \times 8 \times 64$ unit cells. 


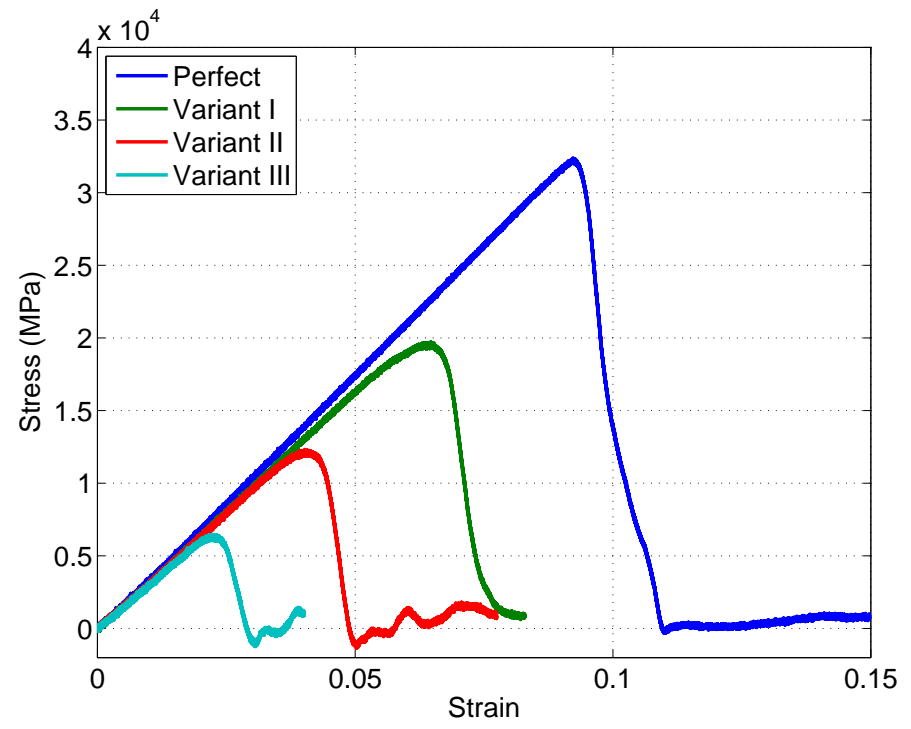

Figure 24: Stress-strain response at $\dot{\epsilon}_{z z}=1.0 \times 10^{9} \mathrm{~s}^{-1}$ for Variants I, II, and III as well as for a perfect PPTA crystallite $(8 \times 8 \times 64$ unit cells) using REAXFF. 

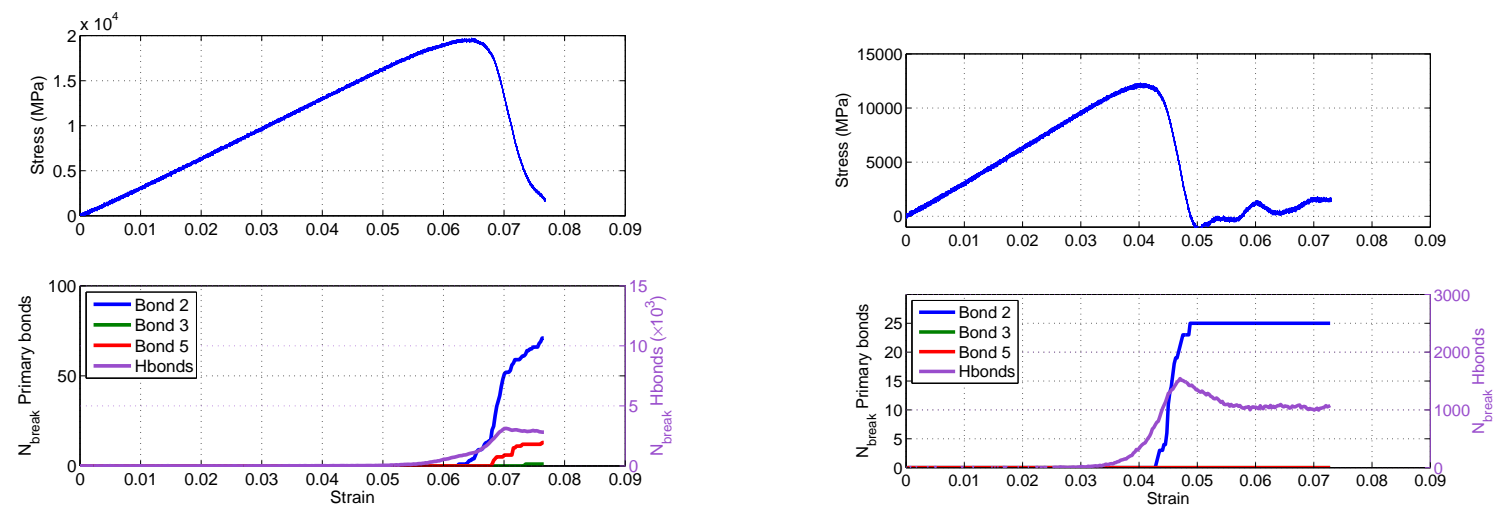

(a) Variant I

(b) Variant II
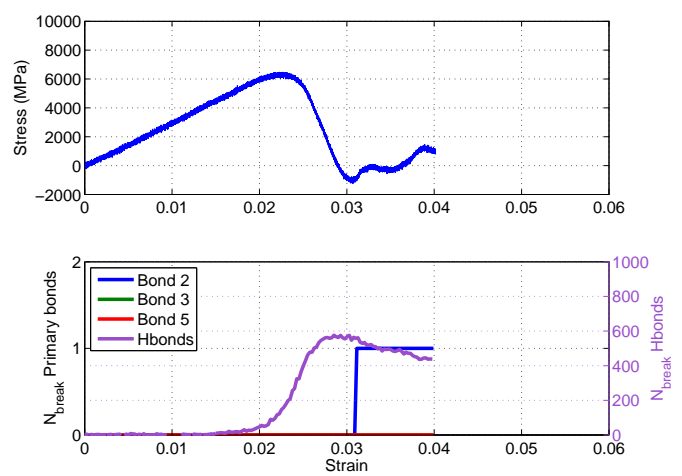

(c) Variant III

Figure 25: Stress-strain and bond break histories for Variants I-III modeled with $\mathrm{REAXFF}$ at a strain rate $\dot{\epsilon}_{z z}=1.0 \times 10^{9} \mathrm{~s}^{-1}$ 


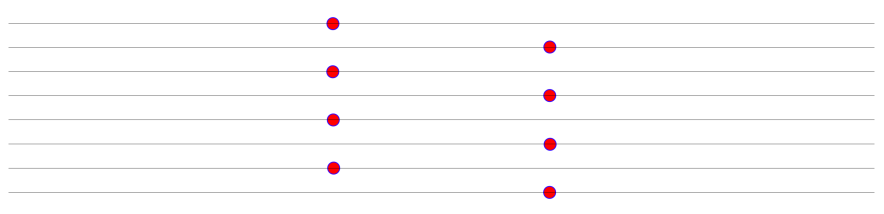

Figure 26: A single sheet of a crystallite with defects in a zig-zag pattern spaced $\pm N$ monomers relative to the bisecting plane of the sheet. 


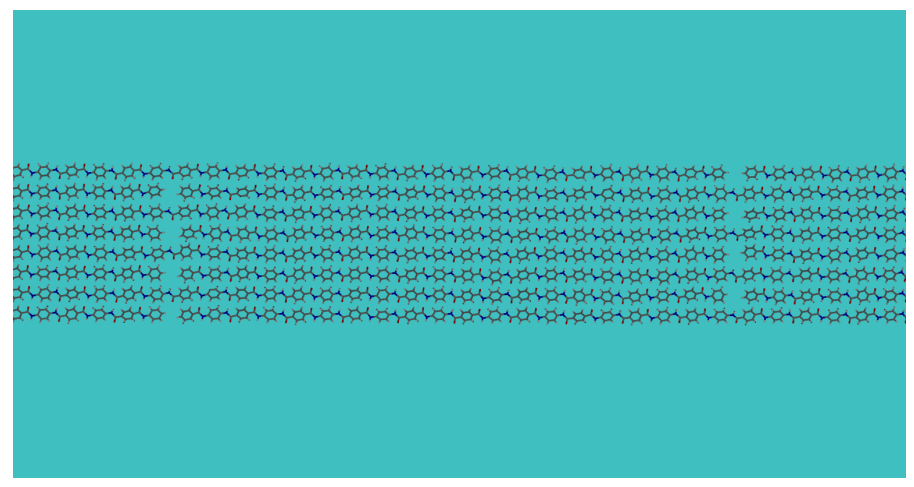

(a) $\epsilon_{z z}=2.0 \%$

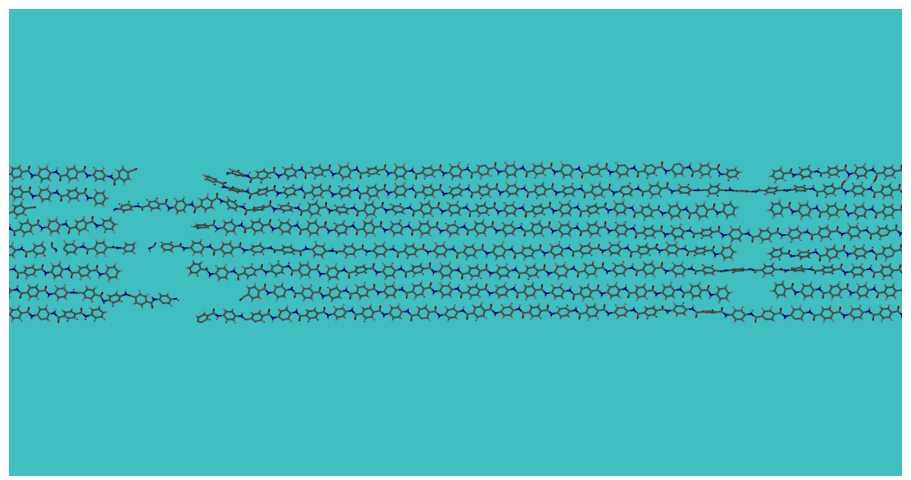

(b) $\epsilon_{z z}=6.0 \%$

Figure 27: Failure of the defect pattern in Figure 26 for $N=5$ using REAxFF. 


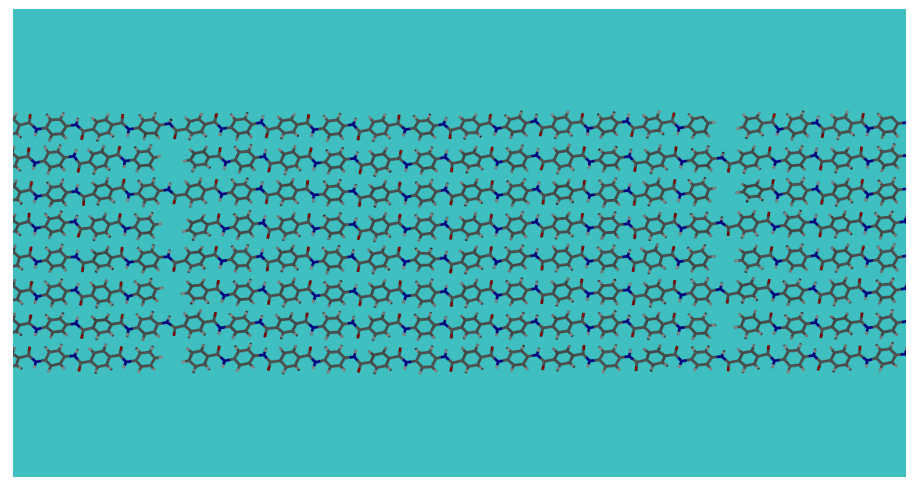

(a) $\epsilon_{z z}=2.0 \%$

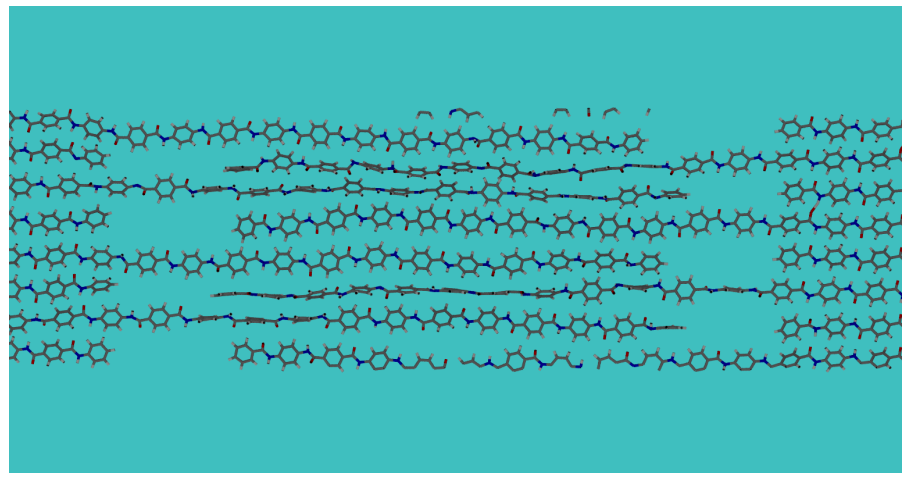

(b) $\epsilon_{z z}=6.0 \%$

Figure 28: Failure of the defect pattern in Figure 26 for $N=3$ using REAxFF. 


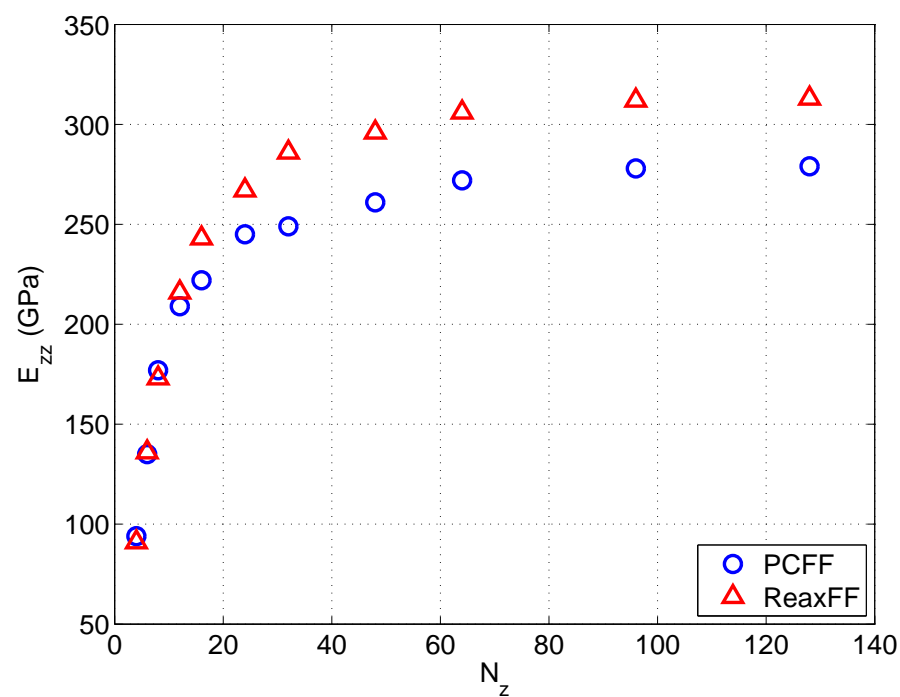

Figure 29: Modulus versus $N_{z}$ for both PCFF and REAxFF. 


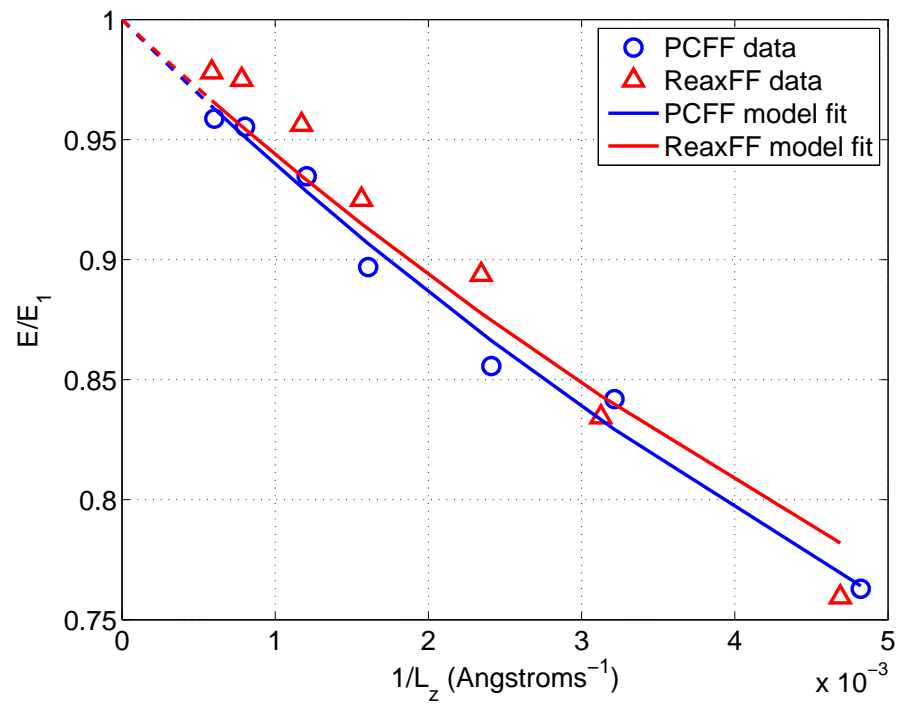

Figure 30: Normalized crystal modulus versus inverse of chain length for both PCFF and REAxFF. The curves represent a best fit of the parameter $\phi$ of equation (6) to the data. 